\title{
Single-arm double-mode double-order planar waveguide interferometric sensor
}

\author{
Summary of Research \\ Principal Investigators: Dr. Sergey S. Sarkisov
}

Period: 1 August 1996 to 31 October 2000

Alabama Agricultural and Mechanical University

Office of Research and Development

4900 Meridian St., P.O. Box 1057, Normal, Alabama 35762

Telephone: /256/851-5675, Fax: /256/851-5030

NASA Grant NAG3-1956 
A. Principal Investigator

Dr. Sergey S. Sarkisov

B. Research Faculty

1. Dr. Martha Reddy

2. Dr. Michael Curley

C. Graduate Students involved

1. Curtis Banks

2. Darnell Diggs

D. Undergraduate students

Andre Taylor

Wallace Lee II

Okedi Omeriji 


\section{Table of Contents}

1. Objectives and Accomplishments 4

$\begin{array}{lr}\text { 2. Motivation } & 8\end{array}$

$\begin{array}{lr}\text { 3. Theory } & 10\end{array}$

4. Material Selection $\quad 19$

$\begin{array}{lr}\text { 5. Experimental Setup } & 29\end{array}$

$\begin{array}{ll}\text { 6. Experimental results } & 30\end{array}$

$\begin{array}{ll}\text { 6.1. Heating cycle } & 30\end{array}$

6.2 Chemical Sensing $\quad 32$

$\begin{array}{ll}\text { 7. Discussion } & 37\end{array}$

$\begin{array}{ll}\text { 8. Polyimide based high temperature sensor } & 38\end{array}$

9. Design of a compact sensor $\quad 40$

10. Conclusions 41

11. References $\quad 42$

Appendix A. List of publications related to the project 44

Appendix B. List of graduate students supported by NASA Grant NAG3-1956 46 


\section{Objectives and Accomplishments}

The goals and objectives of the project in the initial proposal have been formulated as follows:

1-st Year (1997-1998)

1. Material selection. Materials for the waveguide interferometer will be selected from high temperature polyimide resins doped with thermally stable organic compounds. For specific extra high temperature applications, materials will be selected from the glasses prepared by solgel technique and/or possibly from ion-implanted monocrystals.

2. Thin film waveguide fabrication. Fabrication technique will include spin coating, UV curing, and/or ion implantation.

3. Thin film waveguide characterization. This phase of research will include modal spectroscopy based on prism coupling and propagation loss measurement with an experimental set-up based on imaging with digital CCD camera.

4. Experiments with novel single-arm dual-mode interferometry set-up. Optical thin film slab waveguides will be studied with optical set-up similar to that described in the proposal (Fig. 1 of the proposal). Testing the interferometer as a temperature and pressure sensor for the gaseous combustion products of aeropropulsion systems will be conducted. The gases to be tested will include $\mathrm{CO}, \mathrm{CO}_{2}, \mathrm{NO}, \mathrm{NO}_{2}$, and possibly others.

2-Nd Year (1998-1999)

1. Delineation of optical channel waveguides and their characterization.

2. Fabrication of optical coupling elements including gratings and inlets/outlets for optical fibers.

3. Testing the interferometer sensors in an optical channel waveguide configuration.

3-Rd Year (1999-2000)

1. Development of purged gas chamber and precise heater for testing single-arm interferometric sensors.

2. Testing the waveguide coupled with optical fiber lines. 
The following accomplishments have been achieved during the first year:

1. Material selection. Metal substituted phthalocyanines were selected and studied as potential indicator dyes that can be used in the waveguide sensor.

2. Design and construction of the experimental purged gas chamber. The experimental set-up included an additional mixing chamber, which allowed exposure to the sensor to a certain gas mixture for a short period and to characterize the time response. It also included vacuumsealed multimode fiber line, which delivered light to and from the sensor inside the chamber to external signal processing equipment.

3. Preliminary study of the effects of gases adsorbed by polymeric waveguide on its refractive index. The obtained results showed that the refractive index is sensitive to the presence of the gases and that the single-arm double-mode interferometry could be used in gas sensing. However, quantitative characterization of the interferometric waveguide sensor could be accomplished only after finishing construction of the gas chamber.

First accomplishment met the first goal established for the first year. Goals 2 and 3 were met when the experimental planar waveguide structure was fabricated for preliminary experiments. The fabrication technique was spin coating. Characterization included prism coupling to determine waveguide thickness and refractive index. Propagation losses could be determined using digital imaging of the trace of a propagating mode. Accomplishment 3 met the goal 4 established for the first year. We did some preliminary experiments showing that the refractive index of the waveguide reacts to the presence of atmospheric gases.

Preliminary experiments also showed the importance of quantitative characterization of the sensor response to various gases such as $\mathrm{CO}_{2}, \mathrm{NH}_{3}, \mathrm{NO}$, and $\mathrm{NO}_{2}$. A purged gas chamber built during the third year could perform the characterization. Taking into account all possible delays associated with the construction of the chamber, design and construction was started during the first year. $90 \%$ of this work was done. Accomplishment 2 met the goal 1 for the third year. The construction had to be completed by the end of 1997. During 1998, testing and characterization of the sensor in the chamber was planned. This could provide more realistic environment such as variable concentration of gases mixed at various proportions, presence of water vapor, and elevated temperature (up to $250^{\circ} \mathrm{C}$ ).

The following accomplishments have been achieved during the second year: 
1. Material selection: The films of PMMA doped with various indicator dyes were studied in the experimental gas chamber. The films doped with Bromocresol Purple and Bromothymol Blue, being exposed to $5 \%$ ammonia in pure nitrogen at 600 Torr, exhibited an irreversible increase of optical absorption within a $100-\mathrm{nm}$ band with the center at $400 \mathrm{~nm}$ accompanied by an increase of the refractive index at $633 \mathrm{~nm}$ of 3.4 to $3.5 \times 10^{-3}$.

2. Design and construction of the breadboard prototype of the sensor: The prototype was coupled to a He-Ne laser light source by a single mode optical fiber line and to a receiving detector by a multimode optical fiber line. The coupling was done with special adjustable prism couplers, which could be selectively placed in contact with the sensing film at any point.

3. Preliminary characterization of the breadboard prototype: The prototype showed sensitivity to the temperature change of $9.37^{\circ} \mathrm{C}$ per $2 \pi$-phase shift, which was equivalent to sensitivity to the index change of $10^{-3}$ per $2 \pi$-phase shift. This indicated that the sensor was capable of detecting gaseous agents, which were under investigation, particularly, ammonia.

It was more relevant to final goals of the project to substitute the 1-st goal for the second year (delineation of optical channel waveguides and their characterization) with the continuation of the material search for the proposed sensor. The first accomplishment met this objective. We obtained films based on PMMA doped with Bromocresol Purple and Bromothymiol Blue that were optically sensitive to ammonia and $\mathrm{NO}_{2}$.

The second achievement met the second objective for the second year. We chose adjustable prism couplers for connecting the sensor to optical fiber lines in our design of a breadboard prototype of the sensor. These couplers had good coupling efficiency at relatively low cost comparing to any other alternatives such as grating couplers.

The third accomplishment met the third objective for the second year. We performed testing the breadboard prototype of the sensor using heating as a technique of changing its refractive index. The only difference was that we ruled out the channel waveguides as irrelevant to the final goals of the project. The feasibility of the sensor could be shown for the slab waveguide configuration without usage of relatively expensive technologies of channel waveguide delineation. 
Since some minor changes in the goals for the first and the second years took place, the following objectives were proposed for the third year of the project.

1. To finish search for appropriate materials for the sensor. The search will be focused on polymer films doped with indicator dyes.

2. To test the breadboard prototype of the sensor in the test gas chamber. The chamber had to be equipped with vacuum feed-through connector for a single mode fiber guiding the light from the remote laser light source to the sensor.

3. To make design of a compact prototype of the sensor.

4. To estimate figures-of-merit of the sensor and make conclusions on its feasibility.

\section{To make summary of research.}

The following accomplishments have been achieved during the third and fourth (no-cost extension) years of the project:

1. Search for materials. We have selected two polymers: poly (methyl methacrylate) and polyimide Ultradel 9020D from Amoco doped with Bromocresol Purple (BCP) as indicator dye for detection and measuring concentration of ammonia in ambient atmosphere. Polyimide is a high-temperature material. We have found that the operating temperature of $\mathrm{BCP}$ doped polyimide film can be as high as $150^{\circ} \mathrm{C}$.

2. Test of the breadboard prototype. We have done an extensive testing of the prototype at elevated temperature and under exposure to gases such as $\mathrm{NH}_{3}, \mathrm{CO}_{2}, \mathrm{NO}$, and $\mathrm{NO}_{2}$ at various levels of humidity. The sensitivity of the sensor to the change of temperature was determined as $1.5^{\circ} \mathrm{C}$ per one full oscillation of the signal. The sensitivity of the sensor to ammonia was 200 ppm per one full oscillation.

3. Design of the compact sensor. Design of the compact sensor was based on integration of coupling prisms with it as discussed below.

4. Figures-of-merit. The sensitivity of the sensor to the change of temperature was determined as $1.5^{\circ} \mathrm{C}$ per one full oscillation of the signal. The sensitivity of the sensor to ammonia was $200 \mathrm{ppm}$ per one full oscillation.

5. Summary of research. The summary of research is given in this document. 
The detailed description of the accomplishments during the final term of the project is provided below.

\section{Motivation}

Optical environment sensors have recently became a fast expanding technology. ${ }^{1,2}$ There are some features of these sensors that attract potential users. The features include simplicity, compactness, ruggedness, robustness, and endurance to electro-magnetic interference, which lack in their traditional electric counterparts. However, reaching higher sensitivity remains an issue for optical sensors. That is where optical interferometric sensors might be helpful, since they have successfully demonstrated for years their great sensitivity in such applications as precise positioning, tracking, and strain measurements. One particular type of such sensors makes use of thin film planar waveguide interferometers. These devices have their thin film light guiding element opened to an ambient medium. Such configuration makes them especially useful for detecting the presence of various chemical and biological agents. Currently, existing interferometric waveguide sensors comprise dual-arm systems with an external reference arm ${ }^{3}$. Also there are dual-arm device with an internal reference arm such as integrated Mach-Zehnder interferometer in various configurations ${ }^{4,5}$. Single-arm systems, which employ interference of different waveguide modes also are used. ${ }^{6-13}$ Single-arm interferometers are distinguished by their simplicity and low cost: they can be virtually implemented in any thin film slab waveguide without sophisticated means of splitting and recombining of signal and reference arms or lithographic patterning, as in case of integrated optical Mach-Zehnder system. Conventional single-arm devices in slab waveguide configuration use two cross-polarized modes $\mathrm{TE}_{\mathrm{i}}$ and $\mathrm{TM}_{\mathrm{i}}$ 
of the same order $i^{9 \cdot 13}$ They are sometimes called "polarimetric sensors". The sensing effect is based on a difference between the propagation constants of the modes, which produces a phase shift between them at the end of the waveguide. When the environment changes, the difference between the propagation constants and consequently the phase shift also undergoes a change. The interference of the cross-polarized modes decoupled from the waveguide can be implemented by projecting their optical field vectors into the same polarization plane. This converts the change of the phase shift into variation of intensity. However, the difference between the propagation constants of $\mathrm{TE}_{\mathrm{i}}$ and $\mathrm{TM}_{\mathrm{i}}$ modes of the same order $i$ does not change rapidly enough to utilize the full potential of optical interferometry in achieving great sensitivity.

We present a novel single-arm interferometer with improved sensitivity. The interferometer uses modes of different orders. The difference between the propagation constants of the modes changes more rapidly in this case. The double-order waveguide interferometer can generally use the combinations of equally polarized $\left(\left(\mathrm{TE}_{\mathrm{i}}, \mathrm{TE}_{\mathrm{j}}\right)\right.$ or $\left.\left(\mathrm{TM}_{\mathrm{i}}, \mathrm{TM}_{\mathrm{j}}\right)\right)$ or cross polarized $\left(\left(\mathrm{TE}_{\mathrm{i}}, \mathrm{TM}_{\mathrm{j}}\right)\right.$ or $\left.\left(\mathrm{TM}_{\mathrm{i}}, \mathrm{TE}_{\mathrm{j}}\right)\right)$ modes of different orders $i$ and $j$. The focus of the paper is to demonstrate feasibility of the proposed interferometer as a chemical sensor, particularly sensor of gaseous species such as ammonia in ambient air. Beside that we analyze the response of the interferometer to the change of ambient temperature and compare it to theoretical predictions. Since the interferometer is sensitive to both the presence of ammonia and the change of the temperature, we also discuss how to separate these two responses. We use theoretical simulations to show that the double-order interferometer has much greater sensitivity than the polarimetric one. We describe our efforts toward building and characterizing an experimental prototype of chemical sensor of ammonia based on the proposed interferometer. The discussion and 
conclusions on the performance of the prototype and further improvements will then be presented.

\section{Theory}

We derive basic equations and describe numerical simulations of the reaction of the proposed interferometer to the environment-related change of the refractive index of the waveguide. This is done in comparison with conventional single-order polarimetric device.

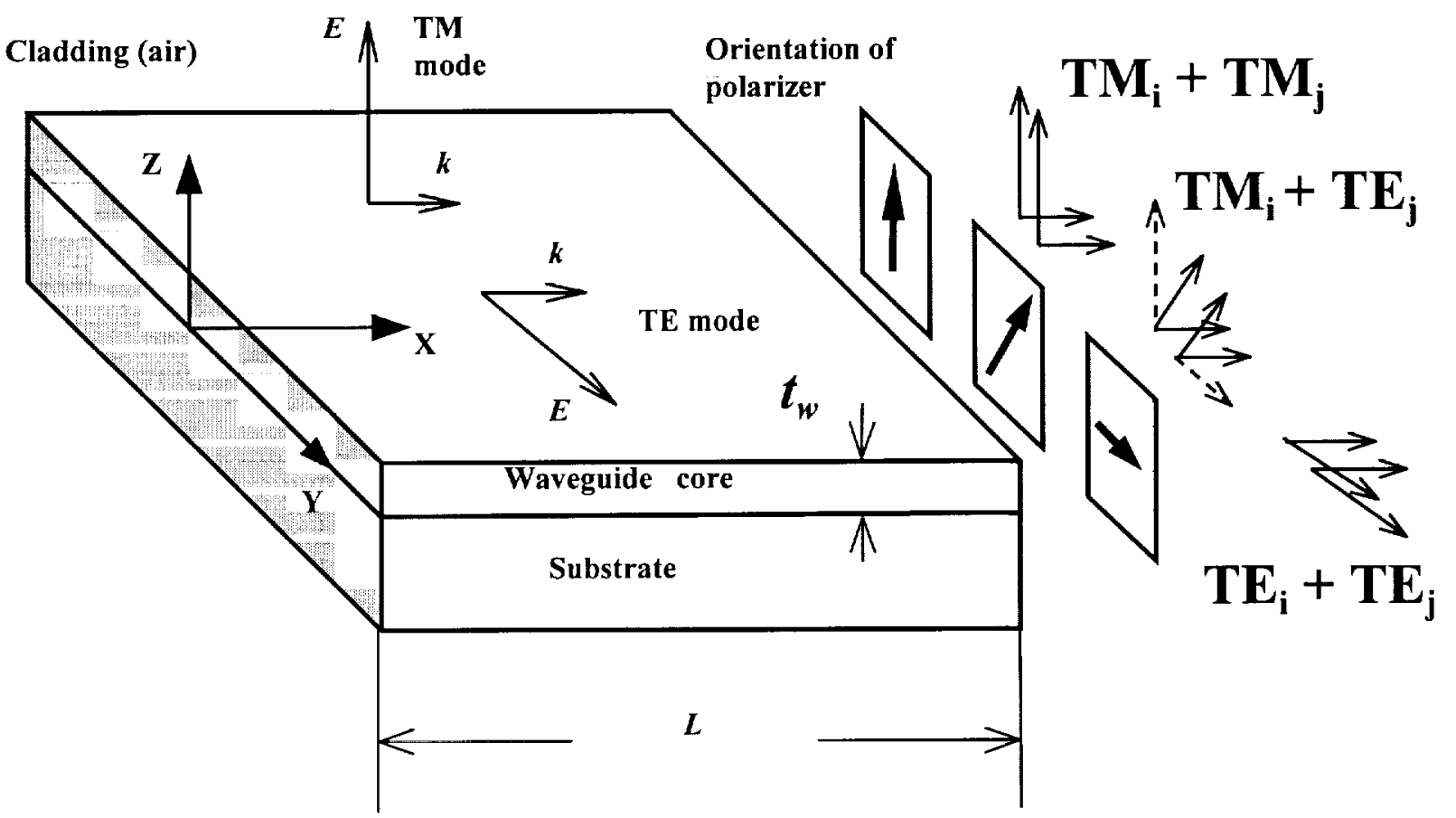

Fig. 1. Sketch of an asymmetric planar slab waveguide that is used as a single-arm interferometric sensor

We consider a slab waveguide, which supports propagating modes of orders «i $»$ and «j»». The waveguide is made as a three-layer structure consisting of a substrate, a polymer film (core), and air cladding as it is shown in Fig. 1. The relation between the refractive indices of the 
waveguide core, substrate and cladding is respectively $n_{W}>n_{S}>n_{\mathcal{C}} \approx 1$. The proposed interferometer is actually a device reading the environment-related changes of differences between propagation constants of modes of different orders. In the case of sensing gaseous species propagation, constants can change due to a variety of effects. We consider the most significant change the refractive index $n_{w}$. Further discussion on this issue will be provided in the material section. The other parameters of the waveguide are assumed to remain constant. An optical wave propagates through the waveguide along $\mathrm{X}$-axis and is assumed infinite in the $\mathrm{Y}$ direction (slab waveguide approximation). Waveguide modes with TE or TM polarization can be combined in three possible ways in order to make up a single-arm interferometer (Fig. 1, right side). We have compared the performance of the interferometer, which employs modes $\mathrm{TM}_{\mathrm{i}}$ and $\mathrm{TM}_{\mathrm{j}}$ (single-arm double-order interferometer), with that of a conventional polarimetric device. The latter one used cross-polarized modes of the same order $\mathrm{TE}_{\mathrm{i}}$ and $\mathrm{TM}_{\mathrm{i}}$ (polarization plane of the output polarizer is oriented at $45^{\circ}$ with respect to the waveguide plane). The dispersion equation for TM mode of a slab waveguide can be written as ${ }^{14}$

$$
\gamma_{w}^{(m)} t_{w} n_{w}^{-1}=\tan ^{-1}\left[\left(n_{w} / n_{s}\right)^{2}\left(\varphi_{s}^{(m)} / \gamma_{w}^{(m)}\right)\right]+\tan ^{-1}\left[\left(n_{w} / n_{c}\right)^{2}\left(\varphi_{c}^{(m)} / \gamma_{w}^{(m)}\right)\right]+\pi m,
$$

where

$$
\begin{aligned}
& \gamma_{w}^{(m)}=k\left(n_{w}^{2}-N_{m}^{(T M)^{2}}\right)^{1 / 2}, \\
& \varphi_{s}^{(m)}=k\left(N_{m}^{(T M)^{2}}-n_{s}^{2}\right)^{1 / 2}, \\
& \varphi_{c}^{(m)}=k\left(N_{m}^{(T M)^{(2)}}-n_{c}^{2}\right)^{1 / 2},
\end{aligned}
$$

and $t_{w}$ is the thickness of the waveguide core, $m$ is the order of the mode, $k=2 \pi / \lambda$ is the wave number ( $\lambda$ is the wavelength of light), $N_{m}(T M)$ is the effective propagation index (propagation 
constant) of the mode. Eq. (1) defines implicitly parameter $\gamma_{w}{ }^{(m)}\left(n_{w}\right)$ as a function of the variable refractive index $n_{w}$. However, Eq. (1) does not have analytical solutions with respect to $\gamma_{w}{ }^{(m)}\left(n_{w}\right)$. It was solved numerically at $m=0$ and $\mathrm{m}=1$ for a waveguide made of a 2-micron thick film of poly (methyl methacrylate) (PMMA) on a fused quartz substrate $\left(n_{S}=1.457\right)$ with index $n_{w}$ assumed to vary in a range from 1.490 to 1.492 . The wavelength of light was $633 \mathrm{~nm}$ (He-Ne laser). With $\gamma_{w^{(m)}}^{(m}\left(n_{w}\right)$ known, we then extracted TM mode indices $N_{0}^{(T M)}=N_{0}^{(T M)}\left(n_{w}\right)$ and $N_{1}^{(T M)}=N_{1}^{(T M)}\left(n_{w}\right)$ using the equation derived from Eq. (2)

$$
N_{m}^{(T M)}\left(n_{w}\right)=\left[n_{w}^{2}-\frac{\gamma_{w}^{(m)^{2}}\left(n_{w}\right)}{k^{2}}\right]^{1 / 2} .
$$

The difference between propagation constants of the modes is generally a nonlinear function of index $n_{w}$. However, near a certain initial value $\widetilde{n}_{w}$ it could be expanded in Taylor series with respect to small parameter $n_{w}-\widetilde{n}_{w}$ as

$$
\begin{aligned}
N_{0}^{(T M)}\left(n_{w}\right)-N_{1}^{(T M)}\left(n_{w}\right) & =R_{0}+R_{1}\left(n_{w}-\widetilde{n}_{w}\right)+R_{2}\left(n_{w}-\widetilde{n}_{w}\right)^{2}+\ldots, \\
& \approx R_{0}+R_{1}\left(n_{w}-\widetilde{n}_{w}\right),
\end{aligned}
$$

where $R_{i}$ are the coefficients of polynomial expansion. With respect to $n_{w}-\widetilde{n}_{w}$, we truncated the Taylor expansion to the term of the first order of magnitude in the second line of Eq. (6).

The phase shift between two modes at the exit point of the waveguide was obtained as

$$
\begin{aligned}
\Delta \Phi\left(n_{w}\right) & =k L\left[N_{0}^{(T M)}\left(n_{w}\right)-N_{1}^{(T M)}\left(n_{w}\right)\right], \\
& \approx \frac{2 \pi L}{\lambda} R_{0}+\frac{2 \pi L}{\lambda} R_{1}\left(n_{w}-\widetilde{n}_{w}\right), \\
& \approx \frac{2 \pi L R_{1}}{\lambda} n_{w}+\Delta \Phi^{\prime}\left(\widetilde{n}_{w}\right),
\end{aligned}
$$


where $L$ is the distance, which both modes propagate through the waveguide, $\Delta \Phi^{\prime}\left(\tilde{n}_{w}\right)=2 \pi L\left(R_{0}-R_{1} \tilde{n}_{w}\right) / \lambda$ is the part of the phase shift, which does not depend on variable $\operatorname{index} n_{w}$.

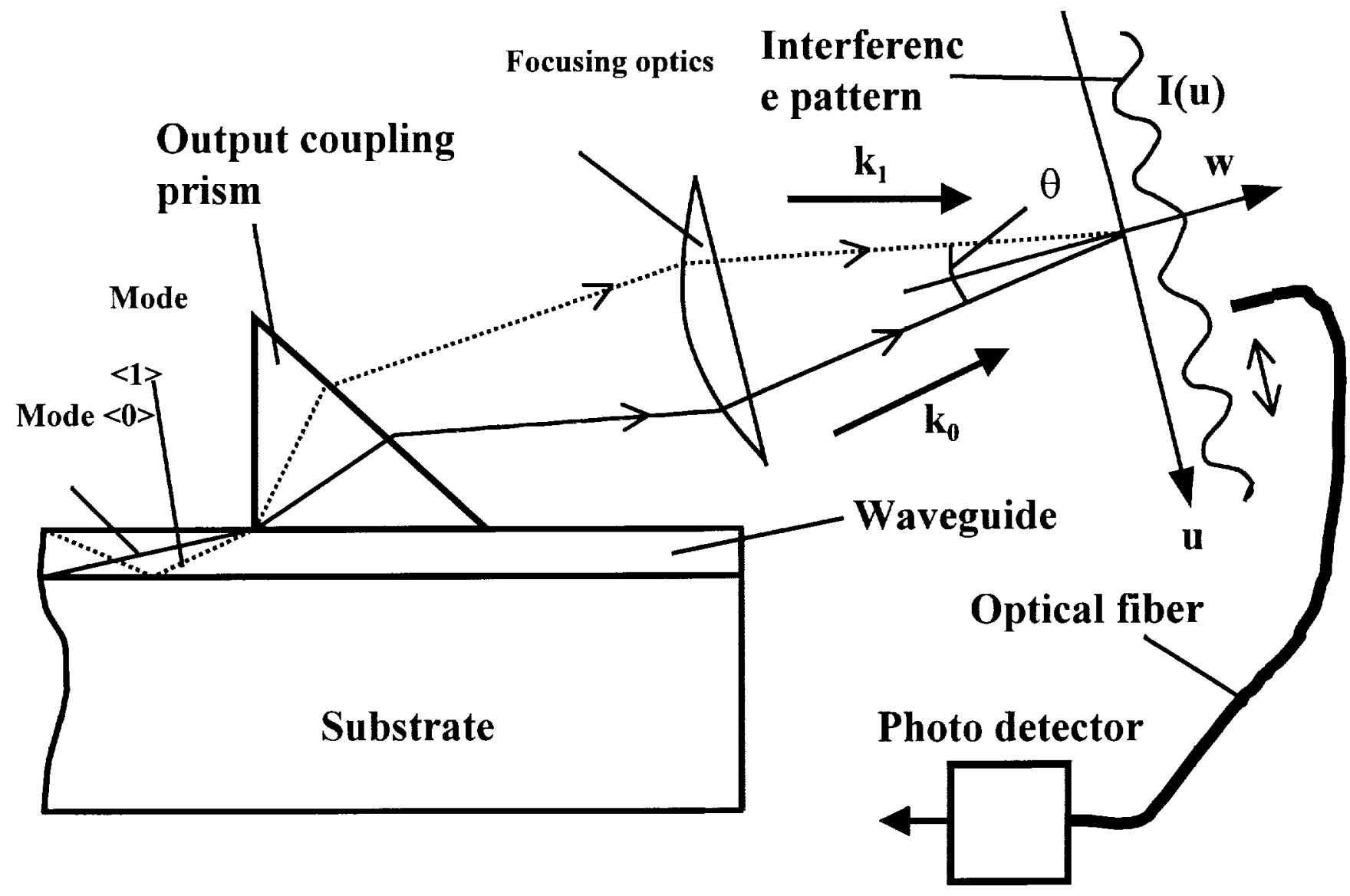

Fig. 2. Diagram illustrating the technique of recombining modes in single-arm, double-mode, and double-order interferometer.

We then assumed that the modes were decoupled from the waveguide into two coherent free propagating waves. This can be done with an output-coupling prism as illustrated in Fig. 2. Mode « $0 »$ in Fig. 2 corresponds to $\mathrm{TM}_{0}$ and mode «1»- to $\mathrm{TM}_{1}$. The waves are recombined 
again by a focusing optics producing a fringe interference pattern in a distant plane. The coordinate system associated with the plane is defined by radius vector $\mathrm{r}=(u, v, w)$, where $w=0$ at the plane. The optical field of the wave corresponding to mode $« 0 »$ in some location $(u, v)$ in the plane can be described as

$$
E_{0}(u, v)=A_{0} \exp \left[\omega t-\vec{k}_{0} \vec{r}+\Delta \Phi\left(n_{w}\right)+\Delta \Phi_{0}\right]
$$

where $A_{0}$ is the amplitude, $\omega$ is the frequency, $t$ is the time, $k_{0}=\left(k_{u}, k_{v}, k_{w}\right)=(-k \sin (\theta / 2), 0$, $k \cos (\theta / 2))$ is the propagation vector of the wave, $\theta$ is the angle between the propagation directions of two waves (normal to the plane bisects angle $\theta$ ), $\Delta \Phi_{0}$ is a constant phase shift, which might be occasionally accumulated during the propagation of the wave toward the plane. Similarly, the optical field of the wave corresponding to mode «1» can be described as

$$
E_{1}(u, v)=A_{1} \exp \left(\omega t-\vec{k}_{1} \vec{r}\right)
$$

where $A_{I}$ is the amplitude, $k_{I}=(k \sin (\theta / 2), 0, k \cos (\theta / 2))$ is the propagation vector. An optical fiber can be placed in the location $(u, v)$ picking up the light and transmitting it to a photo detector. The photo detector will measure the intensity of the optical field, which is the sum of the optical fields of both waves. Using Eqs. (7) through (9) we derived the following formula for the intensity of the interference pattern in point $(u, v)$ :

$$
\begin{aligned}
I\left(n_{w} ; u, v\right) & =\left\langle\left(E_{0}+E_{1}\right)\left(E_{0}^{*}+E_{1}^{*}\right)\right\rangle \\
& =A_{0}^{2}+A_{1}^{2}+2 A_{0} A_{1} \cos \left[\Delta \Phi\left(n_{w}\right)+K u+\Delta \Phi_{0}\right] \\
& \approx A_{0}^{2}+A_{1}^{2}+2 A_{0} A_{1} \cos \left[2 \pi \frac{n_{w}}{D_{n}}+\Delta \Phi^{\prime \prime}\left(\tilde{n}_{w}, u\right)\right]
\end{aligned}
$$


where $\langle\ldots\rangle$ means time averaging performed by the photo detector, $\left(^{*}\right)$ means complex conjugation, $K=2 k \sin (\theta / 2), D_{n}=\lambda /\left(L R_{1}\right)$ is the parameter, which has the meaning of the period of oscillations of the intensity with respect to the varying index $n_{w}$ and can be referred to as a measure of sensitivity to the change of $n_{w}, \Delta \Phi^{\prime \prime}\left(\widetilde{n}_{w}, u\right)=\Delta \Phi_{0}+K u+\Delta \Phi^{\prime}\left(\widetilde{n}_{w}\right)$ is the part of the phase shift which does not depend on index $n_{w}$. Eq. (10) indicates that the response of the interferometer to environment related variation of $n_{w}$ must be an oscillating light intensity. As one can see, the period of oscillations $D_{n}$ is inversely proportional to distance $L$. The longer the waveguide interferometer, the more sensitive it is.

In order to obtain the response of a single-order polarimetric interferometer employing modes $\mathrm{TE}_{0}$ and $\mathrm{TM}_{0}$, we still had to find out the propagation index of $\mathrm{TE}_{0}$ mode as a function of varying index $N_{0}(T M)=N_{0}{ }^{(T M)}\left(n_{w}\right)$. We followed the procedure similar to that used for the double-order interferometer. The dispersion equation for a TE mode is ${ }^{14}$

$$
\gamma_{w}^{(m)} t_{w}=\tan ^{-1}\left(\varphi_{s}^{(m)} / \gamma_{w}^{(m)}\right)+\tan ^{-1}\left(\varphi_{c}^{(m)} / \gamma_{w}^{(m)}\right)+\pi m
$$

After solving Eq. (11) numerically with respect to $\gamma_{w}{ }^{(m)}\left(n_{w}\right)$ at $m=0$ we obtained $N_{0}{ }^{(T E)}\left(n_{w}\right)$ using equation similar to Eq. (5). We calculated the phase difference between two crosspolarized modes of the same order as

$$
\Delta \Phi_{p}\left(n_{w}\right)=k L\left[N_{0}^{(T E)}\left(n_{w}\right)-N_{0}^{(T M)}\left(n_{w}\right)\right]
$$

The light intensity $I_{p}\left(n_{w}\right)$ in the plane of interference of the modes $\mathrm{TE}_{0}$ and $\mathrm{TM}_{0}$ sent through a $45^{\circ}$-oriented polarizer (as shown in Fig. 1) is given by an equation similar to Eq. (10) but with $\Delta \Phi_{p}\left(n_{w}\right)$ instead of $\Delta \Phi\left(n_{w}\right)$. 


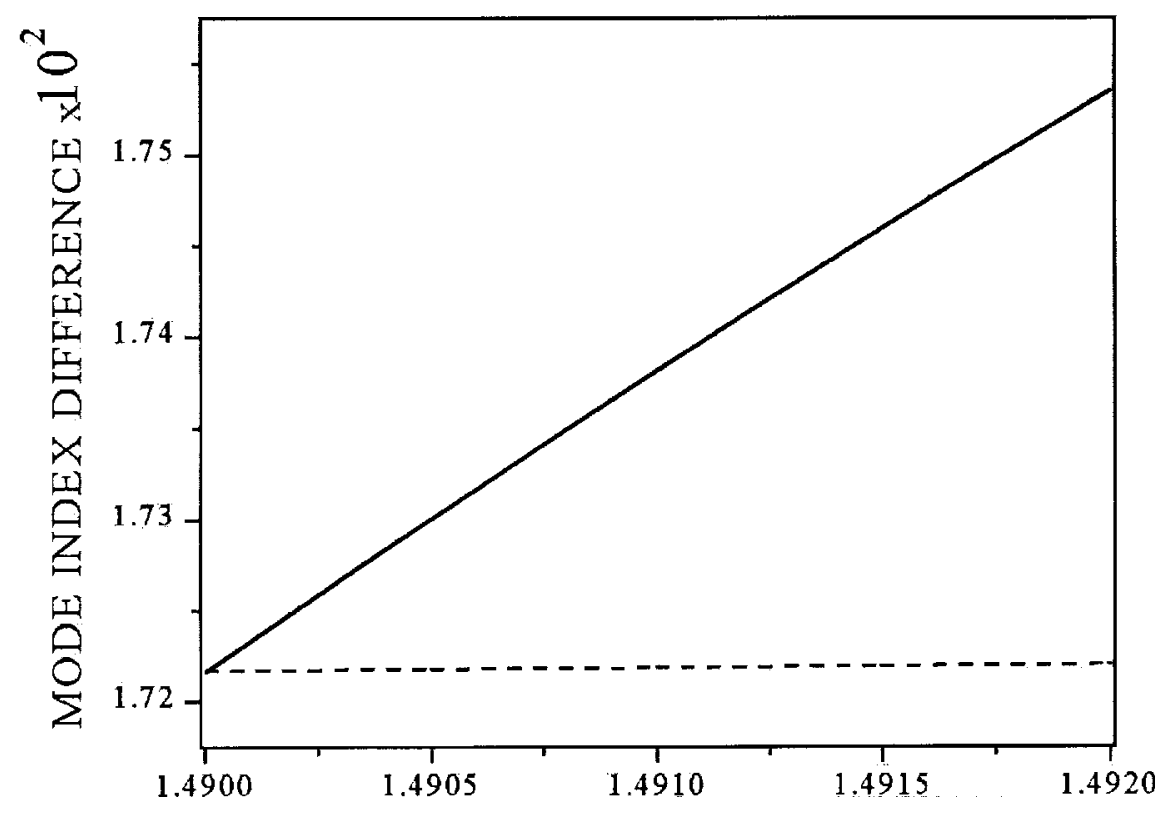

REFRACTIVE INDEX OF THE WAVEGUIDE

Fig. 3. Difference $N_{0}(T M)-N_{I}(T M)$ for a double-order waveguide interferometer (solid curve) and difference $N_{0}(T E)-N_{0}(T M)$ for a single-order polarimetric waveguide interferometer (dashed curve) versus waveguide index $n_{w}$. The calculations were based on the parameters of a 2-micron-thick PMMA waveguide on a fused quartz substrate. The length of the waveguide is 6 $\mathrm{cm}$. In order to simplify comparison, a constant bias of $1.7154325 \times 10^{-2}$ is added to the data represented by the dashed curve.

The difference $N_{0}(T M)\left(n_{w}\right)-N_{l}{ }^{(T M)}\left(n_{w}\right)$ for a double-order interferometer as a function of $n_{w}$ is presented graphically in Fig. 3 together with $N_{0}(T E)\left(n_{w}\right)-N_{0}(T M)\left(n_{w}\right)$ for a singleorder device. We used in the calculations the parameters of a 2-micron thick and 6-cm long PMMA waveguide on a fused quartz substrate. The change of $N_{0}(T M)-N_{l}(T M)$ for the 


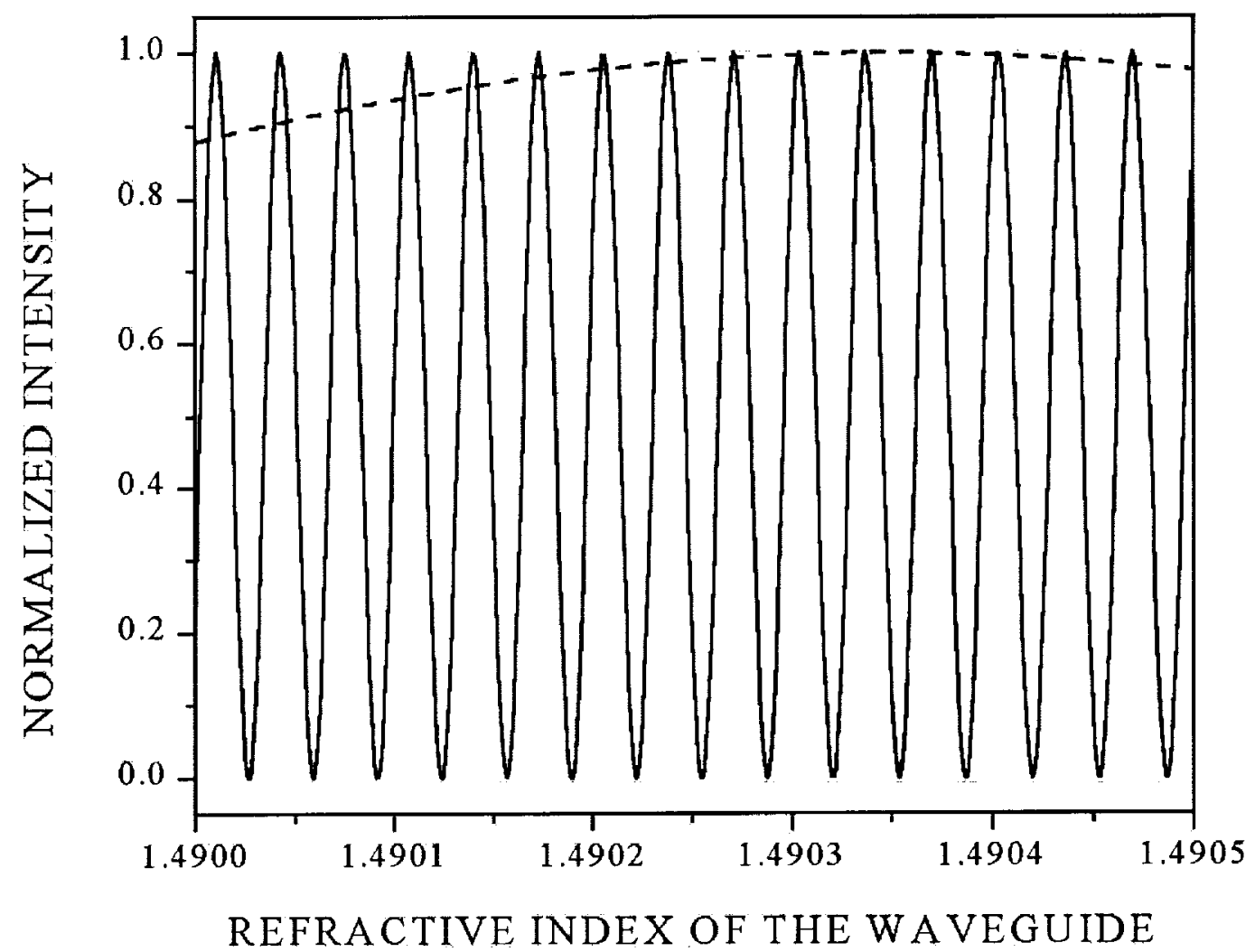

Fig. 4. Normalized intensity versus index for a double-order (solid curve) and a single-order polarimetric (dashed curve) interferometer. Parameters are the same as in Fig. 3.

double-order interferometer is two orders of magnitude greater than the change of $N_{0}{ }^{(T E)}$ $N_{0}{ }^{(T M)}$ for the polarimetric device caused by the same variation of $n_{w}$. Fig. 4 shows the results of the simulations of the light intensity detected at some point of the interferogram generated by double-order and single-order interferometers versus the index of the core. The double-order interferometer generates 15 full oscillations of intensity versus roughly one tenth of full oscillation produced by single-order device per index change of 0.0005 . The period of oscillations of the double-order interferometer $D_{n}$ is approximately 0.000032 . According to the 
comments to Eq. (10), this value can be used as a measure of sensitivity to the change of the index.

We also simulated the response of the proposed double-order sensor to the varying

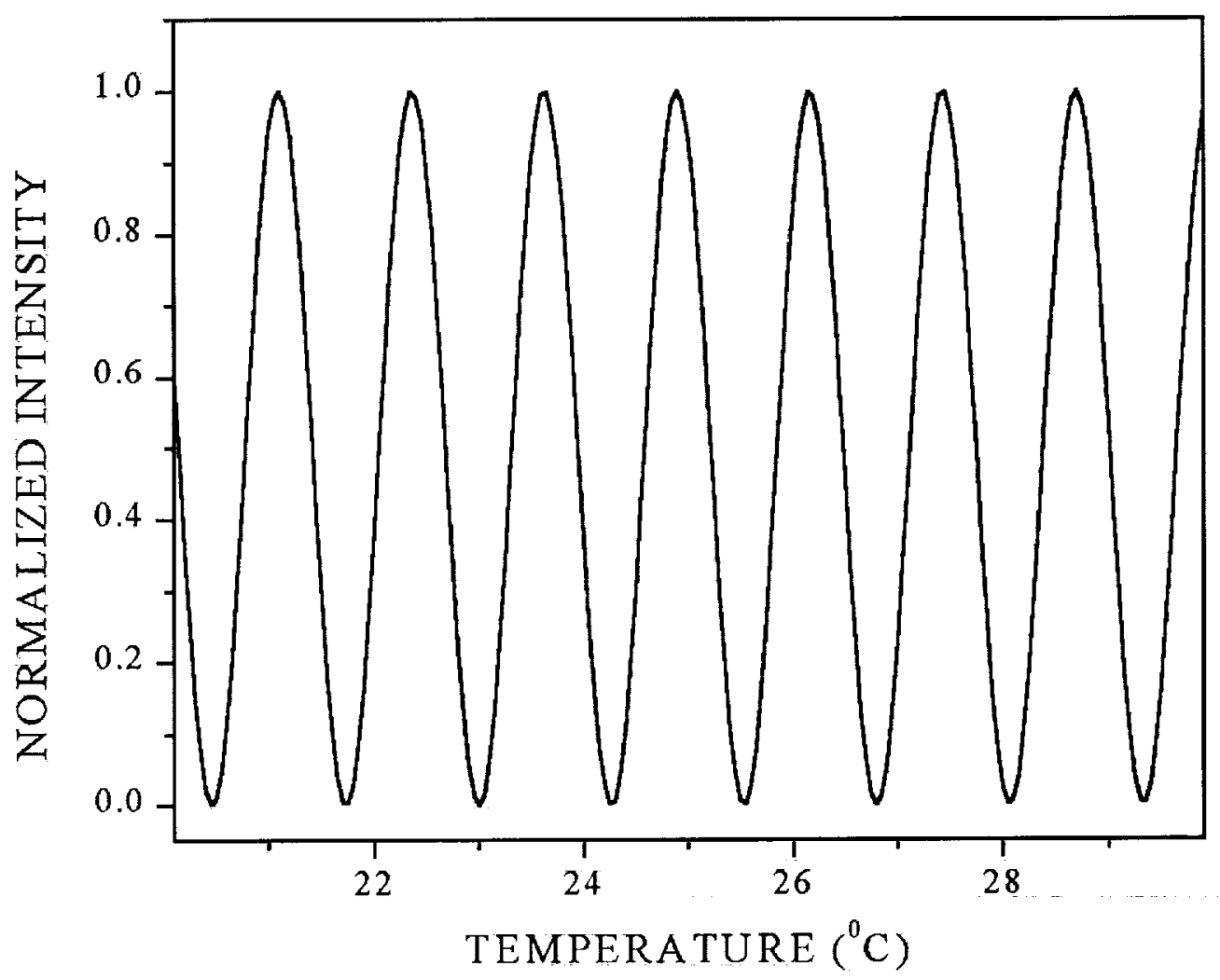

Fig. 5. Normalized intensity versus ambient temperature for a double-order interferometer. Parameters are the same as in Fig. 3.

ambient temperature (Fig. 5). The change of the refractive index $n_{w}$ was calculated using formula

$$
n_{w}\left(t^{0} C\right)=n_{w}\left(t_{i}^{0} C\right)+\frac{\partial n_{w}}{\partial t^{0} C}\left(t^{0} C-t_{i}^{0} C\right),
$$


where $t_{i}{ }^{0} \mathrm{C}$ is the initial temperature of the waveguide, $\partial n_{w} / \partial^{0} \mathrm{C}$ is the temperature coefficient of the refractive index $n_{w}$. Our previous study on heating dye doped PMMA waveguides with laser radiation gave us an estimated value for the temperature coefficient of refractive index $n_{w}$ of nearly $-1.0 \times 10^{-5}$ per ${ }^{\circ} \mathrm{C}^{15}$ We also applied an equation similar to Eq. (13) to the refractive index of the substrate $n_{S}\left(t^{\circ} \mathrm{C}\right)$, which is assumed to be made of fused quartz. The temperature coefficient of the refractive index of fused quartz is close to $9.9 \times 10^{-6}$ per ${ }^{0} \mathrm{C}$ (Ref. 16, p. H-411). Because the waveguide inevitably expands under heating, we also included in our model the thickness of the waveguide $t_{w}$ and its length $L$ as variable parameters:

$$
\begin{aligned}
& t_{w}\left(t^{0} C\right)=t_{w}\left(t_{i}^{0} C\right)\left[1+\beta_{t w}\left(t^{0} C-t_{i}^{0} C\right)\right], \\
& L\left(t^{0} C\right)=L\left(t_{i}^{0} C\right)\left[1+\beta_{L}\left(t^{0} C-t_{i}^{0} C\right)\right],
\end{aligned}
$$

where $\beta_{t w}$ and $\beta_{L}$ are the coefficients of linear thermal expansion of the waveguide core (PMMA, $\beta_{t w} \sim 5.0 \times 10^{-5}$ per ${ }^{\circ} \mathrm{C}$, Ref. 16 , p. H-399) and the substrate (fused quartz, $\beta_{L} \sim 5.5 \times 10^{-7}$ per ${ }^{0} \mathrm{C}$, Ref. 16, p. H-399). Equations (1) through (10) were properly corrected to account for the variation of $t_{w}\left(t^{0} C\right)$ and $L\left(t^{0} C\right)$. As it can be seen from Fig. 5, the response of double-order interferometer to the change of temperature is a typical oscillating function with the period of oscillations close to $1.3^{\circ} \mathrm{C}$. In a similar fashion to what we discussed in the comments to Eq. (10), we can use this period as a measure of the sensitivity to the temperature change.

\section{Material selection}

In order to build a sensor, we had to select a material of waveguide core, which changes its refractive index in response to the presence of a targeted chemical substance, such as ammonia in ambient air. One of the choices was a thin film of polymer doped with an 
appropriate indicator dye. Dye-doped polymers are traditionally used in optical chemical sensors or "optodes" based on change of optical absorption. ${ }^{2}$ In our case, however, the sensitive optical parameter to be measured was not absorbance but refractive index. There is still a connection between the refractive index and the absorption coefficient described by the Kramers-Kronig formula ${ }^{17}$ :

$$
\Delta n_{w}(\lambda)=\frac{c}{\pi} P \int_{0}^{\infty} \frac{\Delta \alpha_{w}\left(\lambda^{\prime}\right)}{\lambda^{2}-\lambda^{\prime 2}} d \lambda^{\prime},
$$

where $\Delta n_{w}$ is the change of the refractive index of the waveguide core, $\lambda$ is the wavelength, $c$ is the speed of light in vacuum, $P$ stands for the principal value of the integral, $\Delta \alpha_{w}$ is the change of the absorption coefficient of the waveguide core. According to Eq. (16), the change of the index at a wavelength $\lambda$ is proportional to an aggregate change of $\alpha_{w}$ within the entire absorption band of the material. Polymer film doped with an indicator dye, which changes its optical absorption in response to ammonia, will change its refractive index at a wavelength outside the absorption band that can be measured by the proposed double-order interferometer. A simplified theoretical description of the chemical response of such sensor can be constructed in the following way.

Assuming that the change of the absorption takes place in a relatively narrow band $\Delta \lambda$ near wavelength $\lambda_{0}$, which is the case for many indicator dyes, we rewrite Eq. (16) as

$$
\begin{aligned}
\Delta n_{w}(\lambda) & \approx \frac{c}{\pi} \frac{\Delta \alpha_{w}\left(\lambda_{0}\right) \Delta \lambda}{\lambda^{2}-\lambda_{0}^{2}}, \\
& =F\left(\lambda, \lambda_{0}\right) \Delta \alpha_{w}\left(\lambda_{0}\right),
\end{aligned}
$$


where $F\left(\lambda, \lambda_{0}\right)$ is the factor of proportionality between the change of absorption and the change of index. The change of absorption can be expressed in terms of the number of molecules of the indicator dye (reagent) reacted with the targeted substance (analyte) (Ref. 2, p. 208) as

$$
\Delta \alpha_{w}\left(\lambda_{0}\right)=\varepsilon\left(\lambda_{0}\right) v,
$$

where $\varepsilon\left(\lambda_{0}\right)$ is the molar optical absorptivity of the reacted dye, $v$ is the number of the reacted dye molecules per unit of volume of the waveguide core. In case of reversible chemical reaction between the analyte penetrating into the waveguide core and the reagent immobilized in it, we have for $v$ in equilibrium (Ref. 2, p. 22)

$$
v=\frac{v_{t} S_{b} C^{\prime}}{1+S_{b} C^{\prime}} \approx v_{t} S_{b} C^{\prime}
$$

where $v_{t}$ is the total number of the dye molecules per unit of volume of the waveguide, $S_{b}$ is the reagent-analyte binding constant, $C^{\prime}$ is the concentration of analyte penetrating into the waveguide. The second short form of Eq. (19) is for the case when either the binding constant or the concentration $C^{\prime}$ is small. We then assume that $C^{\prime}$ is directly proportional to the concentration of analyte in ambient atmosphere as $C^{\prime}=p C$, where $p$ is the permeability factor of the waveguide core and $C$ is the ambient concentration of the analyte. Using Eqs. (17) through (19) we can derive formula for the change of index

$$
\Delta n_{w}(C, \lambda) \approx F\left(\lambda, \lambda_{0}\right) \varepsilon\left(\lambda_{0}\right) v_{1} S_{b} p C .
$$

Eq. (20) thus determines the relation between the concentration of the targeted substance and the change of the refractive index, which can be detected by the proposed interferometer according to the model discussed in the theoretical section. 
However, if the wavelength of the laser source of the interferometer is falling into the absorption band of the indicator dye, the propagating modes of the waveguide will experience an increasing absorption during exposure to the analyte. In this case the model of the sensor described by Eqs. (1) through (10) has to be modified in order to account for absorption in the waveguide core. Getting a dispersion equation for the modes similar to Eq. (1) and solving it must be much more complicated task in this case. However, the effect of absorption can be accounted in rather simple way by introduction of loss indices of the modes in absorbing waveguide. The amplitudes of the modes given by Eqs. (8) and (9) can now be modified as

$$
\begin{aligned}
& E_{0}^{\prime}(u, v)=E_{0}(u, v) \exp \left\{-\sigma_{0}\left[\Delta \alpha_{w}(C)\right]\right\}, \\
& E_{1}^{\prime}(u, v)=E_{1}(u, v) \exp \left\{-\sigma_{1}\left[\Delta \alpha_{w}(C)\right]\right\},
\end{aligned}
$$

where $E_{0}(u, v)$ and $E_{I}(u, v)$ are the amplitudes of the modes given by Eqs. (8) and (9) respectively, $\sigma_{0}$ and $\sigma_{l}$ are the loss indices of the modes depending on absorption change, which is proportional to the concentration of analyte $C$ (see Eqs. (18) and (19)). Assuming the proportionality of the loss indices to the change of absorption, we can obtain

$$
\begin{aligned}
& \sigma_{0} \approx \sigma_{0}(0)+\eta_{0} C, \\
& \sigma_{1} \approx \sigma_{1}(0)+\eta_{1} C,
\end{aligned}
$$

where $\sigma_{0}(0)$ and $\sigma_{l}(0)$ are the loss indices at zero concentration, $\eta_{0}$ and $\eta_{1}$ are the factors of proportionality between the concentration and the loss indices. Taking into account Eqs. (21) through (24), we can rewrite Eq. (10) for the response of the sensor as

$$
I(C ; u, v) \approx\left[A_{0}^{\prime} \exp \left(-\eta_{0} C\right)\right]^{2}+\left[A_{1}^{\prime} \exp \left(-\eta_{1} C\right)\right]^{2},
$$




$$
+2 A_{0}^{\prime} \exp \left(-\eta_{0} C\right) A_{1}^{\prime} \exp \left(-\eta_{1} C\right) \cos \left[2 \pi \frac{C}{D_{C}}+\Delta \Phi^{\prime \prime \prime}(u)\right]
$$

where $A_{0}{ }^{\prime}=A_{0} \exp \left[-\sigma_{0}(0) L\right]$ and $A_{l}^{\prime}=A_{l} \exp \left[-\sigma_{l}(0) L\right]$ are the amplitudes of optical fields, $D_{C}$ $=\lambda / L R_{1} F\left(\lambda, \lambda_{0}\right) \varepsilon\left(\lambda_{0}\right) v_{l} S_{b} p$ is the period of oscillations of the intensity with respect to the change of $C$, which determines the sensitivity of the interferometer as a chemical sensor, $\Delta \Phi^{\prime \prime}(u)$ is the phase shift, which does not depend on the concentration of the analyte. However, a presence of a strong absorption is not a very desirable situation for the sensor, since losses might spoil the oscillating response of the interferometer.

As a waveguide core material and host for the dye dopant we used polymer PMMA, which can be easily dissolved in a number of commercial solvents and mixed with various indicator dyes. It can be also easily processed into a thin film using spin casting. The film of PMMA is permeable by various gaseous agents. As an indicator dye sensitive to ammonia, we selected Bromocresol Purple (BCP), which has been previously used in an absorption-based optical sensor. ${ }^{18}$ As the first step, we investigated the reaction of the optical absorption of the PMMA/BCP system to the presence of dry ammonia. The indicator dye was added to a solution of PMMA in chlorobenzene ( $1 \mathrm{~g}$ solids per $10 \mathrm{ml}$ liquids) at a concentration of $10 \mathrm{mM}$ per liter (5.5\% weight concentration of the dye in PMMA). The solution was filtered with a PTFE filter ( 0.1 -micron pore size). Then it was spin cast at $500 \mathrm{rpm}$ for $120 \mathrm{~s}$ on 1 by 3 -inches fused quartz substrates. The samples were baked in open air at a temperature of $70^{\circ} \mathrm{C}$ for one hour. No visible change in the samples was observed after baking. The final thickness of the PMMA/BCP films was close to 3 micron. The samples were placed one after another into an experimental gas chamber. The first sample was exposed to ammonia. We filled the chamber with ammonia diluted in pure nitrogen to a molar concentration of $5 \%$. The remaining samples were exposed 
separately to $\mathrm{N}_{2}$, and $\mathrm{CO}_{2}, \mathrm{NO}$, and $\mathrm{NO}_{2}$ diluted in pure nitrogen to $5 \%$ concentration. The pressure of gas mixtures during the exposure was maintained at 600 Torr. Within the chamber, each sample was mounted between the terminals of emitting and receiving multi-mode fibers. Each fiber terminal was equipped with a beam collimator in order to reduce losses of light propagating through the sample. The fibers were connected to a Varian Cary $3 \mathrm{E}$ UV-Visible spectrophotometer using a standard fiber optics coupler. In addition, we also measured any possible irreversible or slow recovering change of the refractive index of the samples after exposure. This was done using the prism coupling technique ${ }^{19}$ with a Metricon 2010 prism coupler at $633 \mathrm{~nm}$. The refractive index of the films was of the order of 1.49 and greater than the index of the quartz substrate (1.457). This provided the condition for using the films as slab optical waveguides.

During the exposure to dry ammonia we observed the change of absorption of the PPMMA/ BCP sample. Initially almost colorless film appeared to be yellow after exposure. This corresponded to a rise of absorption in a band from 350 to $450 \mathrm{~nm}$ with a central peak at $400 \mathrm{~nm}$. The change of absorbance was irreversible. The mechanism of this phenomenon is not clear yet. It was accompanied by an irreversible increase of the refractive index at $633 \mathrm{~nm}$ of up to $3.5 \times 10^{-3}$ in agreement with the Kramers-Kronig relation (Eq. (16)). There was also some noticeable irreversible absorption increase near $350 \mathrm{~nm}$ in response to $\mathrm{NO}_{2}$ associated with a refractive index change of the order of $10^{-4}$. The color of the film also turned to yellowish. The samples exposed to other gases did not exhibit any noticeable change of absorption and refractive index.

However, our main focus was on a reversible change of absorption of dye-doped polymer films that could potentially make them attractive to repeatable chemical sensing. It is well known that $\mathrm{BCP}$, being mixed with buffer solution of $\mathrm{NOH}$ in water, exhibits a reversible change of 
color from yellow to purple when $\mathrm{pH}$ changes form 5.2 to 6.8 (slightly acidulous to neutral) ${ }^{20}$ Klein et al. reported similar behavior of $\mathrm{BCP}$ doped into a $\mathrm{SiO}_{2}$ film prepared by a conventional sol-gel technique. ${ }^{18}$ The film had initially yellow color and changed its color to purple after being exposed to ammonia, which acts as an acceptor of proton from the dye and increases $\mathrm{pH}$. The color change was reversible and corresponded to a rise of an absorption peak near $600 \mathrm{~nm}$.

We performed simple exploratory experiments on reversible optical absorption change of a PMMA/BCP thin film sample using a vapor of conventional medical ammonia spirit $(65 \%$ alcohol). The sample was put inside a closed standard 1-cm spectroscopic cell with few drops of ammonia alcohol in it. This produced a saturated vapor of ammonium hydroxide. The cell with the sample in it was placed in a Varian Cary 3E UV-Visible spectrophotometer for continuous monitoring of the absorption spectrum of the sample. Termination of the exposure to the vapor was achieved by opening the cell and ventilating it. Experimental results are shown in Fig. 6 . Curve 1 in Fig. 6 represents the absorption spectrum of a freshly made film of PMMA/ BCP. The fresh film apparently did not have any significant absorption in range 300 to $850 \mathrm{~nm}$. Then the sample was kept for three months in open air. It became slightly yellowish. Its absorption spectrum is represented by curve 2 in Fig. 6 . The absorption gained some irreversible increase in a band from 350 to $450 \mathrm{~nm}$ with a central peak at $400 \mathrm{~nm}$ similar to that of the sample, which we 


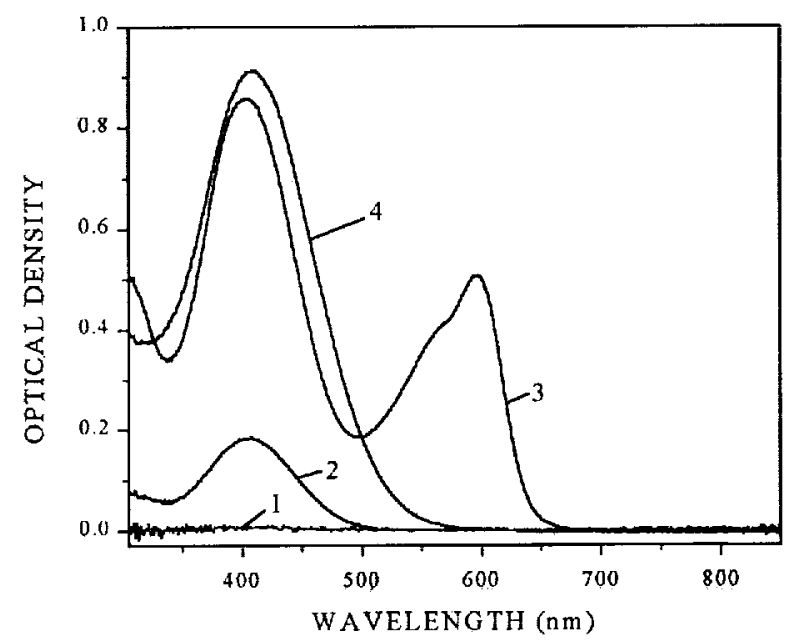

Fig. 6. Optical absorption spectrum of PMMA/BCP film (1) freshly made, (2) three months after making and before exposure to ammonia, (3) after holding into a closed container with saturated vapor of medical ammonia spirit $(65 \%$ alcohol) for 10 minutes, (4) after ventilation of the container for 12 hours. The film had a thickness of 3 micron. It was spin cast on a fused quartz substrate. The concentration of BCP in PMMA host was $7 \%$ by weight $(13.2 \mathrm{mM}$ per liter in initial solution of PMMA in chlorobenzene).

exposed to dry ammonia in the gas chamber. Curve 3 represents the absorption spectrum of the sample kept for 10 minutes in closed spectroscopic cell with ammonia spirit. Visually we could see that in 10 minutes after closing the cell the entire film of PMMA/ BCP changed its color from yellowish to purple. During 10-min period the absorption reached stationary state with no further change. The response of the absorption of the film to the change of concentration of ammonia was almost instantaneous. In fact, a 10 -min period of time was required by ammonia vapor to reach its saturation in the cell. The change of the color was apparently associated with the rise of an absorption peak at $600 \mathrm{~nm}$, which was previously reported in Ref. 18 . However, the peak at $400 \mathrm{~nm}$ also experienced a significant rise. After complete termination of exposure (ventilation of the cell for 12 hours until all ammonia spirit evaporated) the sample turned its color into deep yellow similar to that of the sample being exposed to dry ammonia in the gas 
chamber. The absorption peak at $600 \mathrm{~nm}$ disappeared but the peak at $400 \mathrm{~nm}$ remained almost unchanged (Fig. 6, curve 4). It has been already discussed in the literature ${ }^{18,21}$ that the reversible absorption of BCP and of similar dye Bromothymol Blue (BTB) in polymer matrices at $600 \mathrm{~nm}$ can be associated with the following chemical reactions:

$$
\begin{aligned}
& \mathrm{NH}_{3} \text { (vapor) }+\mathrm{H}_{2} \mathrm{O} \leftrightarrow \mathrm{NH}_{4}^{+}+\mathrm{OH}^{-}, \\
& \text {Dye (yellow) }+\mathrm{OH}^{-} \leftrightarrow \mathrm{Dye}^{-} \text {(purple) }+\mathrm{H}_{2} \mathrm{O}, \\
& \text { Dye }{ }^{-} \text {(purple) }+\mathrm{NH}_{4}^{+} \leftrightarrow \text { H Dye (yellow) }+\mathrm{NH}_{3} \text { (vapor), }
\end{aligned}
$$

Reactions described by Eqs. (26) and (27) take place in the presence of ammonia and reaction in Eq. (28) takes place when ammonia vapor is removed. Presence of water is essential for the reaction in Eq. (26). It can be supplied in the form of water vapor as in our experiment with ammonia spirit. This, however, makes the optical response of the film opened to the influence of uncontrollable atmospheric moisture. In order to avoid this problem, researchers in Refs. 18 and 21 used hydrophilic polymer hosts. The polymer films adsorbed water vapor until the saturation was achieved. In such a way the influence of the atmospheric moisture could be substantially reduced. In our experiments with hydrophobic PMMA films exposed to dry ammonia in the gas chamber, the lack of water was more likely the reason for the absence of a reversible absorption change with the respective rise and fall of the $600-\mathrm{nm}$ peak. The irreversible absorption change with the rise of the $400-\mathrm{nm}$ peak could be apparently associated with a chemical reaction involving the dye, $\mathrm{NH}_{3}$ and PMMA without water. We could not find any discussion of this subject in the literature. It requires further clarification. 


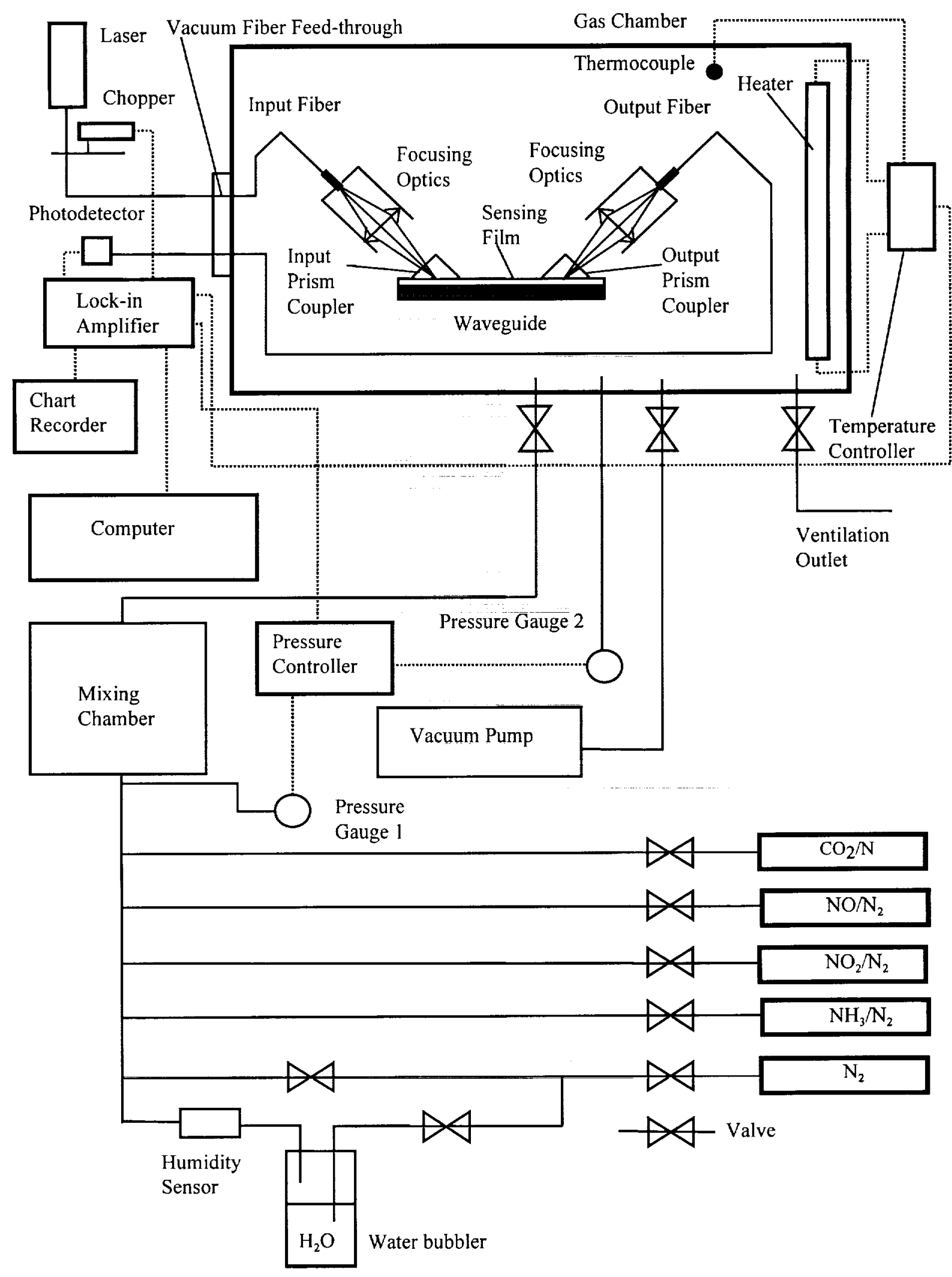

Fig. 7. Experimental set-up for testing single-arm double-mode double-order waveguide interferometric sensor. Dotted lines show electric signal channels. 


\subsection{Experimental set-up}

The schematic of the experimental set-up for testing the concept of a single-arm doublemode double-order sensor is shown in Fig. 7. It includes a gas chamber where the sensor is exposed to various gas mixtures. Gas supply module includes a combination of gas cylinders and a water bubbler connected through valve-controlled pipelines to a mixing chamber. The purpose of the mixing chamber is to prepare a mixture of different gases at a certain proportion before exposing the sensor. The main gas chamber is equipped with an internal heater, thermocouple, and temperature controller. The gas mixture inside the chamber can be heated up to $250^{\circ} \mathrm{C}$. The pressure in the main and mixing chambers is monitored by two Convectron pressure gauges from Granville-Phillips. The light from the external laser source (red He-Ne laser) is sent into the chamber through a vacuum-sealed single mode optical fiber feed-through. An input single mode optical fiber line is coupled to a thin film slab waveguide using focusing optics and a prism coupler. The power of the focusing optics is such that it can excite simultaneously two propagating waveguide modes that will be employed in interferometric sensing. It literally means that the angle of the light cone produced by the focusing system is greater than the separation between the optimum coupling angles of each individual mode.$^{22}$ The coupler is made of Gallium Gadolinium Garnet (GGG) with a refractive index of 1.9648 at $633 \mathrm{~nm}$. A similar approach is used to decouple the light from two propagating modes into the output optical fiber line. Two diverging coherent light beams generated by the modes at the output prism are recombined in the face of the fiber terminal by an output focusing optical system. The beams produce an interference pattern. The spacing between the interference fringes should be several times greater than the diameter of the fiber core. This gives a good contrast to the light intensity oscillations in the sensor response to the environment change. The light collected by the output fiber is sent 
through the second vacuum feed-through fiber line to a photo detector. Signal measurement is done using a light chopper and a lock-in amplifier. The amplifier also receives signals from the thermocouple inside the chamber (temperature) and from the pressure gauges (pressure in the mixing chamber and in the main chamber). The output signal from the lock-in amplifier is recorded with a chart recorder. The lock-in is also connected to a computer through a GPIB interface.

\subsection{Experimental results}

\subsection{Heating cycle}

Preliminary evaluation of the sensor performance was based on its response to the change of temperature. This was done inside the test chamber using the internal electric heater with a PID digital controller Eurotherm 91E. The sensor was a slab waveguide made of a fresh 2micron thick PMMA/BCP film ( $2 \%$ weight concentration of the dye in the polymer or $4 \mathrm{mM}$ per liter of PMMA solution in chlorobenzene). The film was spin cast on a fused quartz substrate at $1000 \mathrm{rpm}$ for $120 \mathrm{~s}$ and baked at $70^{\circ} \mathrm{C}$ for one hour. It was exposed to a $5 \%$ mixture of $\mathrm{NH}_{3}$ and $\mathrm{N}_{2}$ in order to introduce permanent modification of the absorption spectrum at $400 \mathrm{~nm}$. The color of the sample was deep yellow. The waveguide had two propagating modes $\mathrm{TM}_{0}$ and $\mathrm{TM}_{1}$, which we used in the experiment. The travel distance of the modes between the input and output coupling points was $6 \mathrm{~cm}$. The temperature of the ambient air was measured with a chromel-

alumel thermocouple fixed in the middle of the chamber. The signal from the sensor and reading of the thermocouple were digitized by the lock-in amplifier and sent into computer through a 


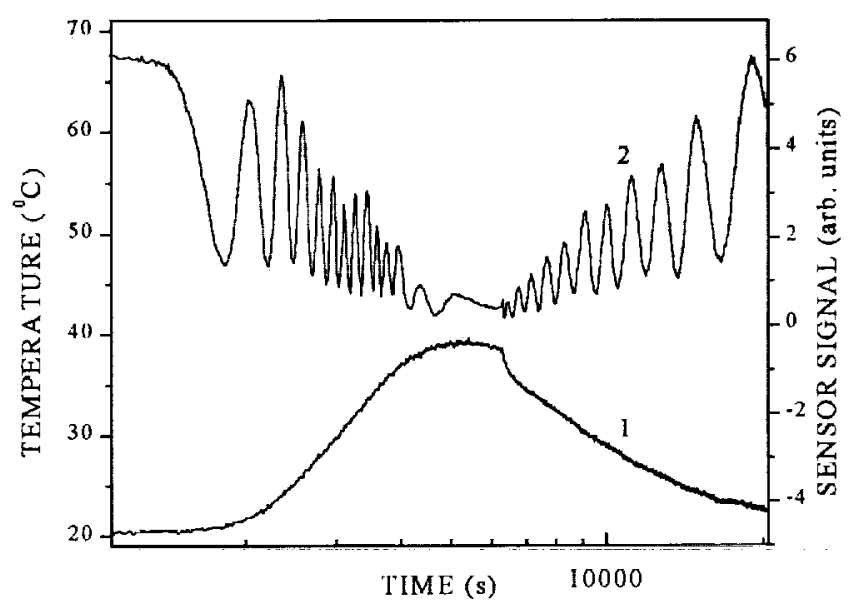

Fig. 8. Temperature (curve 1) and the signal of the sensor (curve 2) during a heating cycle performed inside the experimental chamber without current ramping (rectangular electric current pulse). The sensor is made of a 2-micron thick PMMA/BCP film on a fused quartz substrate.

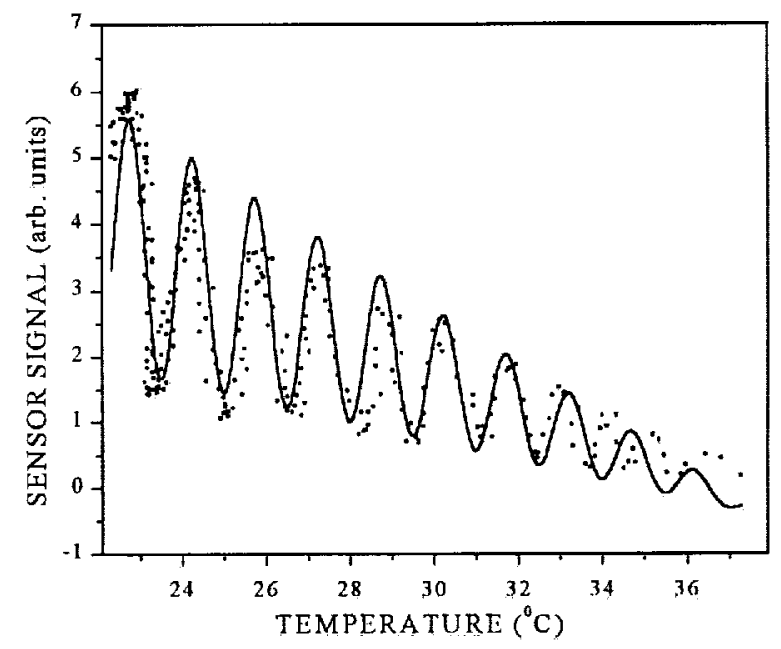

Fig. 9. Signal of the sensor versus temperature for the cooling part of the cycle depicted in Fig. 8. Solid curve represents data fitting.

GPIB data acquisition interface card controlled by a LabVIEW program. Figs. 8 and 9 illustrate the behavior of the sensor in the case when the initial temperature was $20^{\circ} \mathrm{C}$. The heater was set 
to a rectangular current pulse (current ramping disabled), and the stop limit of heating was set at $32^{\circ} \mathrm{C}$. The signal, a typical oscillating wave, exhibited some decay when the temperature went up (Fig. 8, curve 2). This could be attributed to a partial loss of coupling between the waveguide and the prisms due to thermal expansion of the aluminum brackets holding the prisms against the waveguide. There is some discrepancy in signal-versus-temperature readings between heating and cooling parts of the cycle. The reason for this might be the lack of direct contact between the thermocouple and the sensor making impossible to measure instantaneously the actual temperature of the waveguide. We assumed that the thermocouple readings were more close to the actual temperature during the cooling cycle when the thermal equilibrium was more likely reached in the chamber. Based on this assumption, we plotted the signal of the sensor versus temperature in Fig. 9. This dependence could be approximated with function (Fig. 9, solid curve)

$$
I(x)=a-a_{1} x+\left(b-b_{1} x\right) \cos \left(\frac{x}{D_{t}}+c\right),
$$

where $x$ stands for the temperature $t^{0} C, a, a_{I}, b, b_{I}, c$, and $D_{t}$ are the parameters of fitting. Eq. (29) describes periodical oscillations of the sensor readings versus temperature in agreement with the results of the theoretical simulations presented in Fig. 5. It also accounts for the signal decay due to loss of coupling with the help of two terms: $-a_{I^{x}} x$ and $-b_{1} x$. The sensitivity based on the fitting parameter $D$ (period of oscillations) was about $1.5^{\circ} \mathrm{C}$ per $2 \pi$-phase shift (one full oscillation of the signal). The sensitivity is close to $1.3^{\circ} \mathrm{C}$ per $2 \pi$-phase shift, the value obtained from the theoretical simulations.

\subsection{Chemical sensing}


In our experiments on chemical sensing, we used the same sample as in thermal cycling. The target gas was ammonia. The following procedures were used. The sensor was put in the main gas chamber (Fig. 7). Atmospheric air was evacuated from both chambers. Then the chambers were filled with pure $\mathrm{N}_{2}$ to a pressure of 300 Torr. The gas was evacuated again from the mixing chamber. Then the mixing chamber was filled with water vapor from the water bubbler. The concentration of water vapor in the mixing chamber was estimated using the readings of pressure gauge 1 . Then the mixing chamber was filled with a $5 \%$ mixture of $\mathrm{NH}_{3}$ in $\mathrm{N}_{2}$. Ammonia was further diluted in the chamber by filling it with pure $\mathrm{N}_{2}$ to a pressure of near 800 Torr. Based on readings of gauge 1, we estimated the concentration of $\mathrm{NH}_{3}$ in the mixing chamber. The main chamber with $\mathrm{N}_{2}$ at approximately 300 Torr was slowly filled with diluted $\mathrm{NH}_{3}$ leaking from the mixing chamber through a valve connecting the chambers. Pressure readings of gauge 2 gave us an estimate for the concentration of $\mathrm{NH}_{3}$ in the main chamber. The first set of experiments was done with the sample exposed to a mixture of ammonia and wet nitrogen. A typical signal of the sensor changing in time along with the concentration of ammonia is presented in Fig. 10, curves $1 \mathrm{a}$ and $1 \mathrm{~b}$. The initial pressure in the chamber was near 313 Torr. The leak of diluted ammonia stopped when the pressure in both chambers reached equilibrium at a level of 450 Torr. The maximum concentration of the water vapor in the main chamber reached $3651 \mathrm{ppm}$, which corresponded to a relative humidity of $28.4 \%$. Fig. 10 shows an apparent oscillatory behavior of the sensor signal. Though we did not have any accurate means of estimating response time, we could judge that it did not exceed that of the pressure gauge (curve 1a in Fig. 10 represent the concentration of $\mathrm{NH}_{3}$ extracted from the pressure 


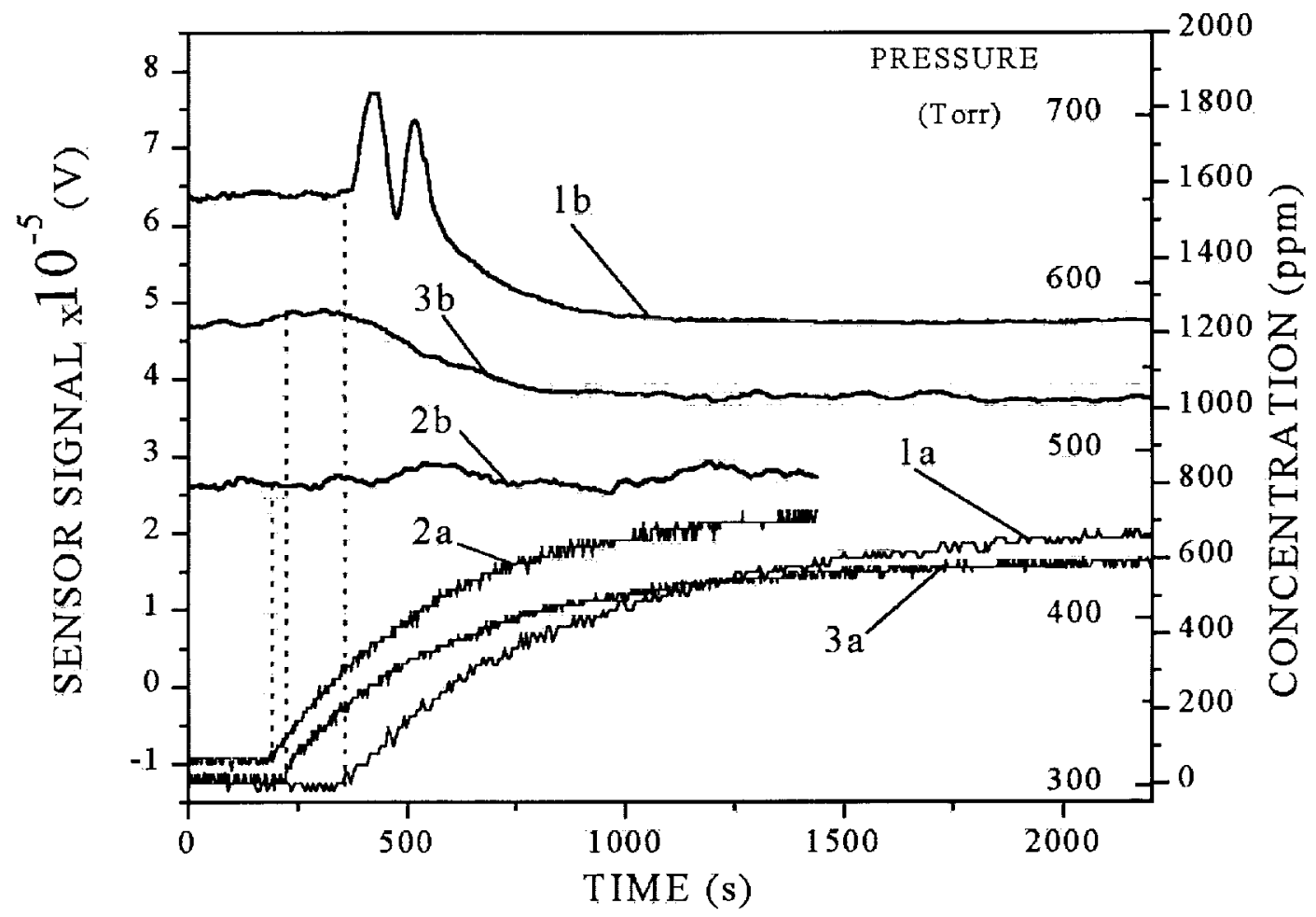

Fig. 10. Kinetics of the response of the sensor to various gas mixtures. Curves $1 \mathrm{a}$ and $1 \mathrm{~b}$ correspond respectively to the concentration of ammonia and to the sensor response in the case when the sensor was exposed to a mixture of ammonia and wet nitrogen (the maximum relative humidity was near $28.4 \%$ ). A value of $4.5 \times 10^{-5} \mathrm{~V}$ is added to actual readings of the sensor in order to make presentation of experimental data by curve $1 b$ more convenient. Curves $2 a$ and $2 b$ correspond respectively to the pressure and to the sensor response in the case when the sensor was exposed to pure nitrogen. Curves $3 a$ and $3 b$ correspond respectively to the concentration of ammonia and to the sensor response in the case when the sensor was exposed to a mixture of ammonia and dry nitrogen. A value of $2.5 \times 10^{-5}$ is added to actual readings of the sensor in order to make presentation of experimental data by curve $3 \mathrm{~b}$ more convenient.

readings). The response time of the sensor (less than $10 \mathrm{~s}$ ) included the time of building up an equilibrium concentration of ammonia within the polymer film and the time of the chemical reactions described by Eqs. (26) through (28). All the changes in the film were reversible. In fact, the sensor was exposed to ammonia hundreds times without anynoticeablepermanent change or 


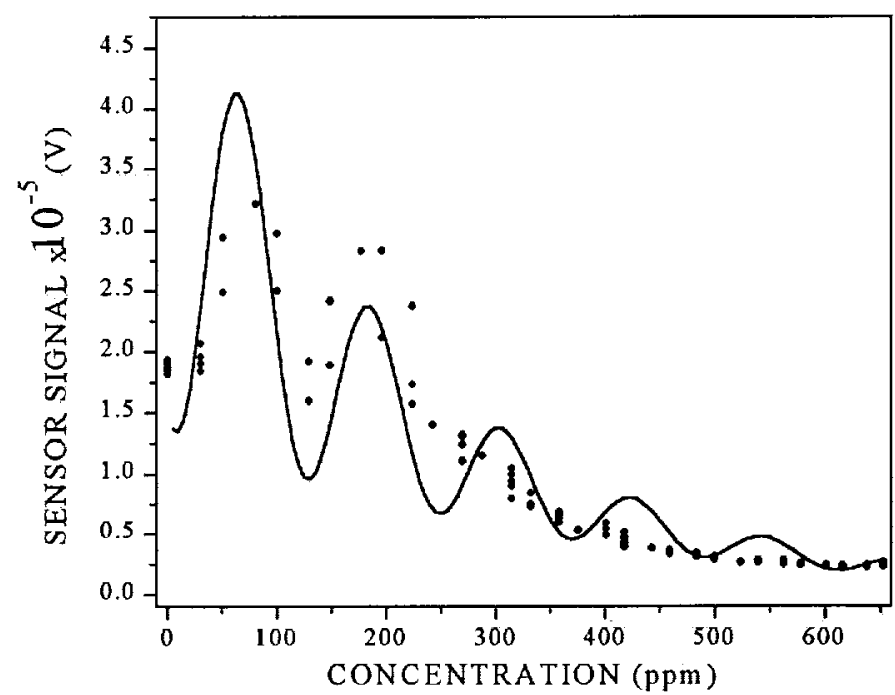

Fig. 11. Signal of the sensor versus concentration of ammonia in wet nitrogen for the experimental cycle depicted in Fig. 10, curves $1 \mathrm{a}$ and $1 \mathrm{~b}$. Solid line represents data fitting.

degradation. The sensor signal is plotted versus concentration of ammonia in Fig. 11. The signal is an oscillating wave with decay. According to the discussion in the material section, the decay could be attributed to absorption of the laser light by the dye. Experimental data could be approximated by the function in Eq. (25), which is plotted as a solid line in Fig. 11. Parameters of fitting are $A_{0} \sim 5.6 \times 10^{-3} \mathrm{~V}^{1 / 2}, A_{1} \sim 1.9 \times 10^{-3} \mathrm{~V}^{1 / 2}, \eta_{0} \sim 2.0 \times 10^{3}, \eta_{I} \sim 3.4 \times 10^{3}, \Delta \Phi^{m \sim} 4.3$. Fitting parameter $D_{C}$ (period of oscillations) gave us an estimate of the sensitivity close to $120 \mathrm{ppm}$ of ammonia per $2 \pi$-phase shift.

Additional experiments were performed in order to prove that the sensor responds to the presence of ammonia, not just to the change of the pressure in the chamber. Curves $2 a$ and $2 b$ in Fig. 10 show the reaction of the sensor to the change of the pressure of pure nitrogen in the chamber. The pressure of $\mathrm{N}_{2}$ went from 310 up to 460 Torr. No significant change of the signal has been detected. We also exposed the sensor to $\mathrm{CO}_{2}, \mathrm{NO}$ and $\mathrm{NO}_{2}$ diluted in $\mathrm{N}_{2}$ without 
detecting a significant response. The sensor has proven to be suitable for selective detection of ammonia.

We also performed some investigation of the role of water vapor in building up the sensor response. Curves $3 \mathrm{a}$ and $3 \mathrm{~b}$ in Fig. 10 show the kinetics of the signal in the case when the sensor was exposed to a mixture of $\mathrm{NH}_{3}$ and dry nitrogen. The reaction of the sensor was apparently much weaker than in the experiments with wet nitrogen (curves $1 \mathrm{a}$ and $1 \mathrm{~b}$ in Fig. 10).

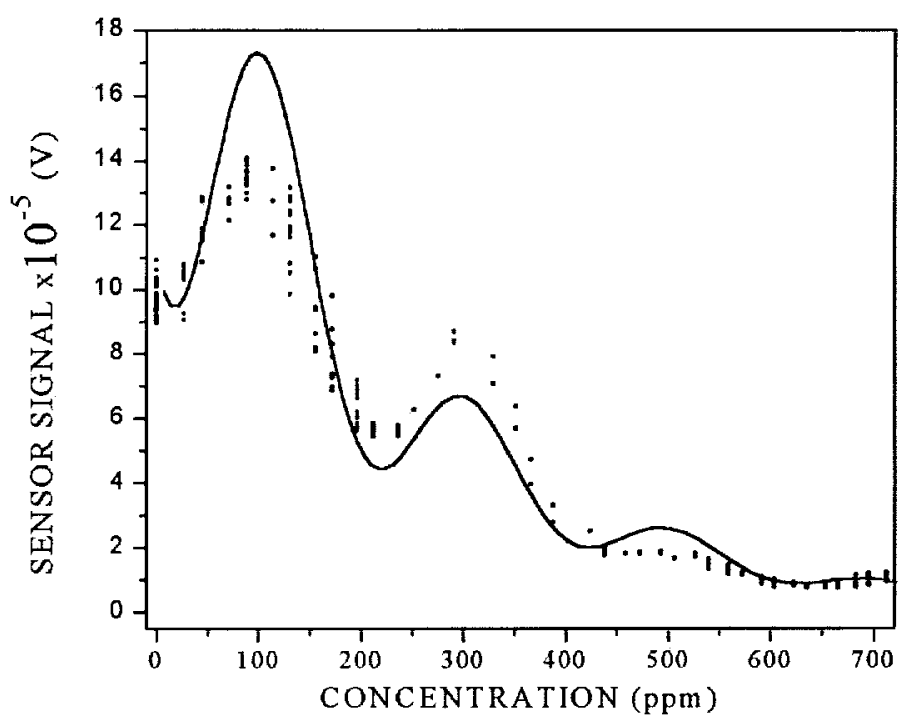

Fig. 12. Signal of the sensor versus concentration of ammonia in the case when the sensor was exposed to a mixture of $\mathrm{NH}_{3}$ and atmospheric air. Maximum relative humidity in the chamber was $98 \%$. Solid line represents data fitting.

The reaction of the sensor to ammonia in atmospheric air is represented by Fig. 12. In this experiment we mixed $\mathrm{NH}_{3}$ with air in the mixing chamber with extra water vapor added and then transferred the mixture into the main chamber being initially filled with air at 300 Torr. The initial relative humidity of the air was $68 \%$. The maximum humidity in the main chamber was approximately $98 \%$. Experimental data in Fig. 12 was approximated by Eq. (25). Parameters of 


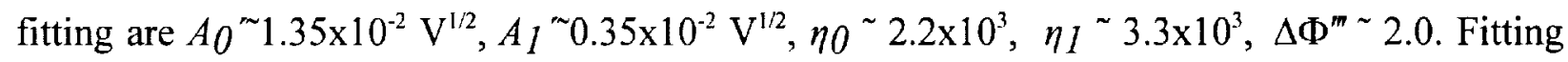
parameter $D_{C}$ (period of oscillations) gave us an estimate of the sensitivity close to $200 \mathrm{ppm}$ of ammonia per $2 \pi$-phase shift.

\subsection{Discussion}

The proposed sensor has demonstrated a selective response to the presence of ammonia and water vapor in ambient atmosphere. The response was an oscillating wave with a decay, which was associated with ammonia-related change of absorption in the waveguide. The sensitivity of the interferometer to ammonia based on the period of oscillations was estimated to be close to $120 \mathrm{ppm}$ per $2 \pi$-phase shift when the ambient atmosphere consisted of pure nitrogen. The sensitivity in air was $200 \mathrm{ppm}$ per $2 \pi$-phase shift. However, ammonia related loss factors $\eta 0$ and $\eta_{l}$ for the modes obtained by fitting experimental data with function in Eq. (25) are almost the same in both cases. It indicates that there might be a chemical or physical mechanism, which inhibits an ammonia-related change of the refractive index of PMMA/BCP film in air at the same level as in pure nitrogen. All this is despite the fact that the change of optical absorption is almost the same. The strong effect of absorption shows that the laser source of the sensor is not optimized: 633-nm wavelength apparently falls into the band of reversible absorption of $\mathrm{BCP}$, which is shown in Fig. 6, curve 3. In order for the sensor to be more suitable for chemical sensing, it has to have more oscillations and less decay for the same concentration of ammonia. The possible solution is switching to a laser source with the wavelength standing away from of the absorption band. It might be, for example, a semiconductor laser emitting in range 675 to 850 $\mathrm{nm}$. Since the absorption effect will be significantly reduced, the film can be loaded with more 
dye without corrupting the performance. According to Eq. (20), larger number of dye molecules per unit of volume of polymer will result in stronger change of the refractive index and correspondingly in more oscillations of the signal per the same concentration of ammonia.

A serious problem was also the influence of moisture on sensor response. Comparing curves $1 \mathrm{~b}$ and $3 \mathrm{~b}$ in Fig. 10 clearly indicates that stronger oscillating signal from the sensor was achieved at higher relative humidity. Following the accomplishments reported in Refs. 18 and 21, we had to use a water saturated polymer matrix instead of hydrophobic PMMA. One of the possible choices could be polymer polyimide, which is currently under investigation.

Heat cycling experiments gave us the sensitivity of the interferometer to temperature change as high as $1.5^{\circ} \mathrm{C}$ per $2 \pi$-phase shift. This is close to $1.3^{\circ} \mathrm{C}$ per $2 \pi$-phase shift obtained from the theoretical simulations (Fig. 5). The temperature change can potentially corrupt the performance of the interferometer as a chemical sensor. This is a common problem of many interferometric sensors, which can be solved by adding a reference temperature-only-sensitive channel. ${ }^{4,5}$ One possible solution is that the additional channel must generate the same response to the ambient temperature while being isolated from chemical exposure by a protecting overcoat. In our case this can be done just by splitting the incident light into two beams, which will be independently injected and decoupled from the waveguide. The reference beam should travel through a protected region of the polymer film.

\section{Polyimide based high temperture sensor}

We have made a high-temperature sensing film using polyimide Ultradel $9020 \mathrm{D}$ from Amoco doped with BCP. The results of the experimental study of the material are presented in Figs. 13 and 14. The material demonstrates very strong reversable absorption near the peak at 
$600 \mathrm{~nm}(13$, curve 2$)$. The reversable absorption remains in place even after keeping the material at $150{ }^{\circ} \mathrm{C}$ for 15 hours and at $200^{\circ} \mathrm{C}$ for 7 hours (Fig. 14, curve 2).

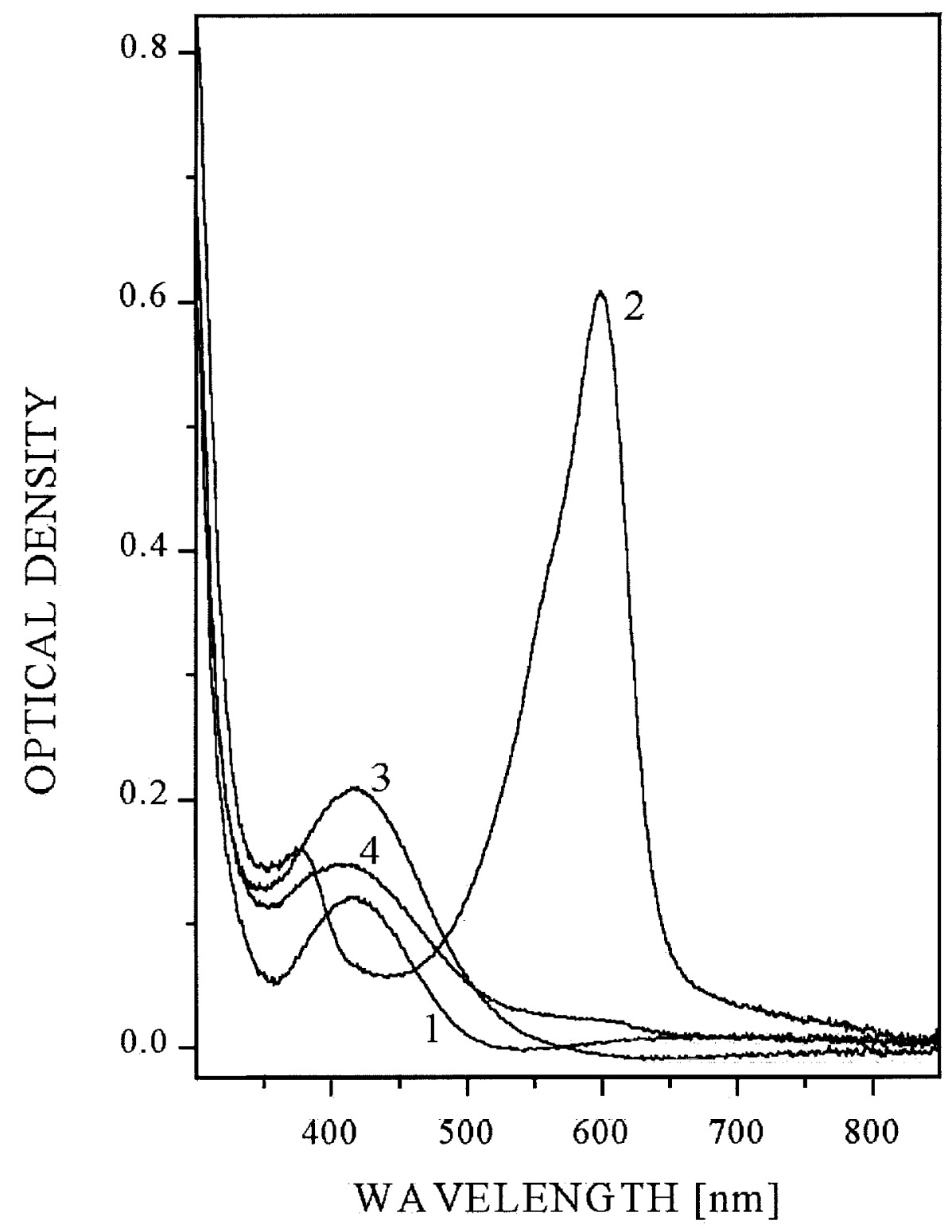

Fig. 13. Optical absorbance of the Bromocresol Purple/Polyimide sample: 1- after making; 2 exposed to ammonia; 3 - after removing ammonia; 4 after being kept at $150^{\circ} \mathrm{C}$ for 15 hours 
The experimental results indicate that the sensor based on this material can operate at $150 \mathrm{C}$ for a significant period of time without losing its sensing capability.

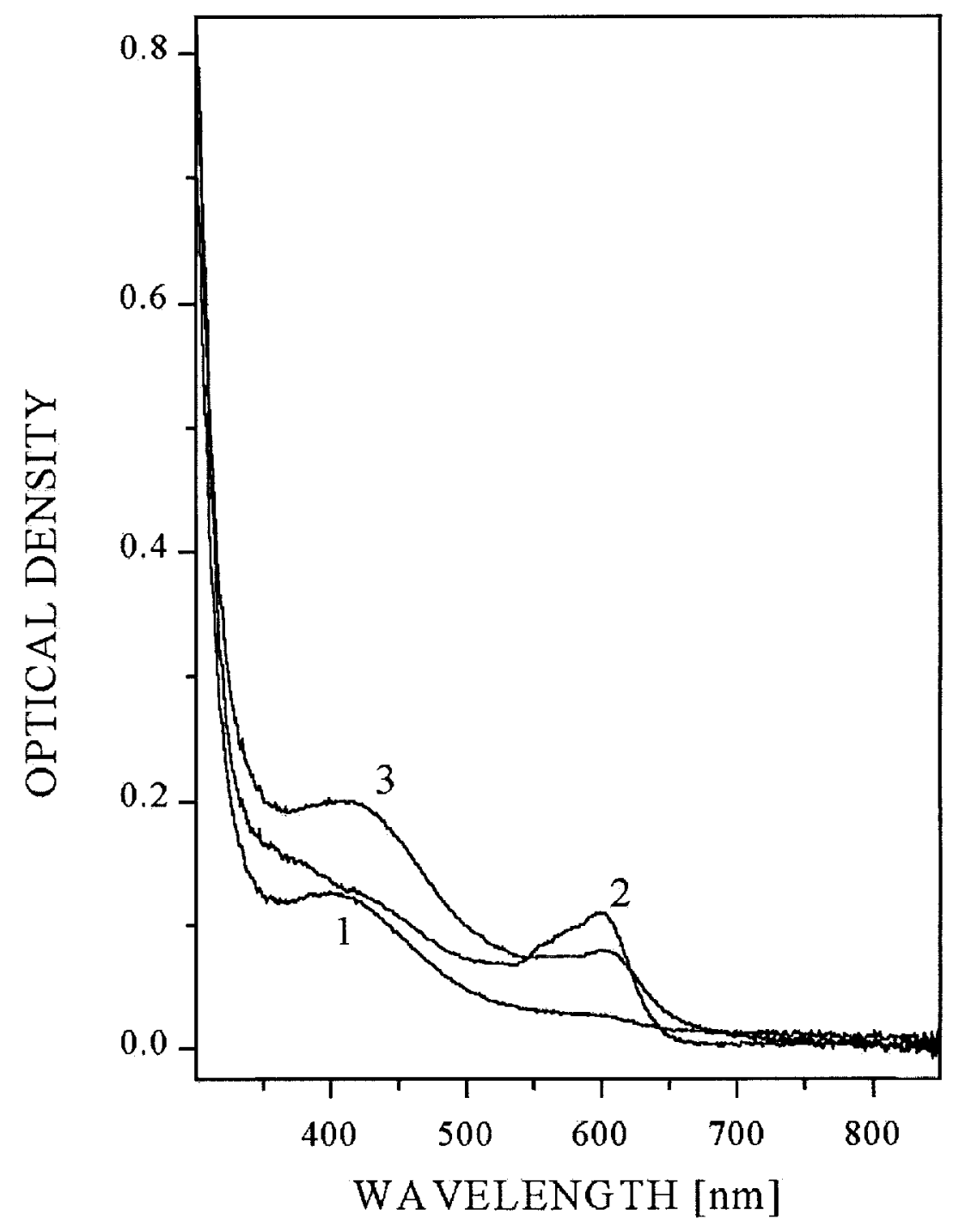

Fig. 14. Optical absorbance of the Bromocresol Purple/ Polyimide sample, which has been baked at $150^{\circ} \mathrm{C}$ for $15 \mathrm{~h}$ and then baked at $200^{\circ} \mathrm{C}$ for $7 \mathrm{~h} \mathrm{(1)} \mathrm{and} \mathrm{then} \mathrm{exposed} \mathrm{to} \mathrm{ammonia} \mathrm{(2)}$ and after ammonia was removed (3). 


\section{Design of a compact sensor}

Design of the compact sensor is presented in Fig. 15. It has two high-index coupling prisms attached to the substrate. The entire polymer film remains open to the air. All the optical

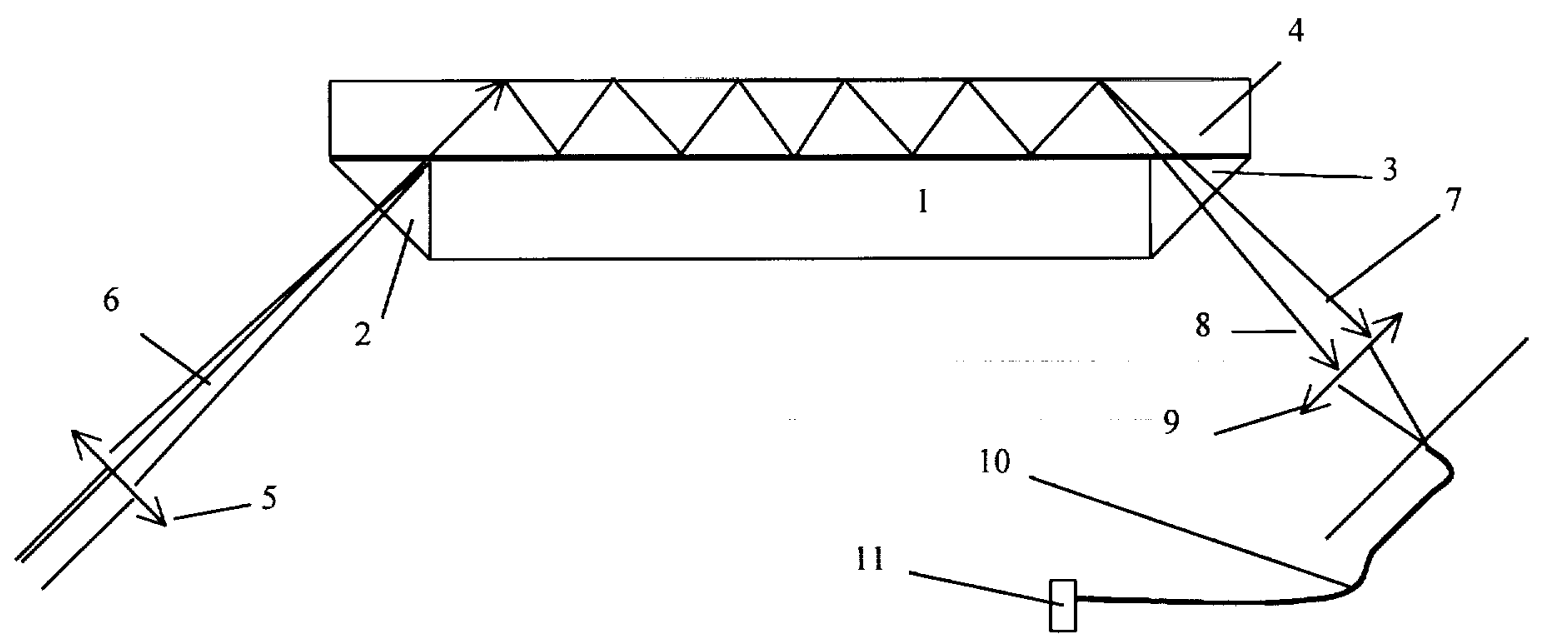

Fig. 15. Thin film single-arm double-mode double-order waveguide sensor integrated with coupling prisms. Element 1 is the fused quartz substrate, 2 is the input coupling prism, 3 is the output coupling prism, 4 is the organic thin film, 5 is the input focusing optics, 6 is the input laser beam, 7 is the beam corresponding to the zero-order mode in the waveguide, 8 is the beam corresponding to the first-order mode in the waveguide, 9 is the output focusing optics, 10 is the optical fiber with input terminal placed in the plane of interference of beams 7 and 8,11 is the photo detector.

parts are located on the back side of the sensor. It makes possible to isolate all the optical parts from gaseous agents while keeping the light guiding film open to them.

We did some initial testing of the proposed design. The high-index prisms made of Galium Gadolinium Garnet (GGG) were attached to a glass substrate as shown in Fig. 16. The film was made of polyimide. One can see a Tm launched into the film and propagating through the film. This demonstrates the feasibility of the proposed design. 


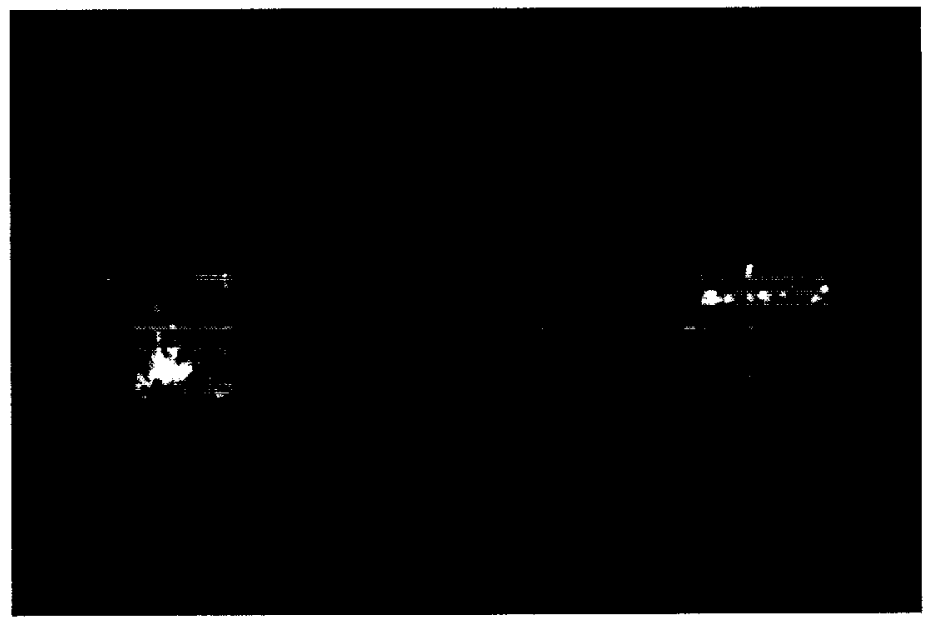

Fig. 16. Experimental thin film waveguide sensor integrated with coupling prisms. A single TM traveling mode is shown.

\section{Conclusions}

We have met the goals stated in section 1 for the project. We have demonstrated the feasibility of a single-arm double-mode double-order waveguide interferometer as a cost efficient alternative to an optical chemical sensor. Experimental prototype was built as a dye-doped polymer waveguide with propagating modes of orders $« 0 »$ and « $1 »$ of the same TM polarization. The prototype demonstrated sensitivity to ammonia of the order of $200 \mathrm{ppm}$ per one full oscillation of the signal. Sensor based on polyimide doped with BCP can operate at elevated temperature up to $150 \mathrm{C}$. Upon the future funding, we are planning to optimize the light source, material and the design in order to achieve sensitivity of the order of $1 \mathrm{ppm}$ per full oscillations.

\section{References}

1. J. Dakin and B. Culshaw, Optical fiber sensors. Vol. 4: Applications, Analysis, and Future Trends (Artech House, Boston, 1997). 
2. G. Boisde and A. Harmer, Chemical and biochemical sensing with optical fibers and waveguides (Artech House, Boston, 1996).

3. K.E. Rochford, R. Zanoni, G.I. Stegeman, W. Krug, E. Miao, and M.W. Beranek, "Pulse-modulated interferometer for measuring intensity-induced phase shifts," IEEE J. Quantum Electron. QE-28, 2044-2050 (1992).

4. N.F. Hartman, J.L. Walsh, D.P. Campbell, and U. Akki, "Integrated optic gaseous $\mathrm{NH}_{3}$ sensor for agricultural applications," in Optics in Agriculture, Forestry, and Biological Processing, G.E. Meyer and J. A. DeShazer, eds., Proc. Soc. Photo-Opt. Istrum. Eng. 2345, 314-323 (1995).

5. N.F. Hartman, J.M. Cobb, and J.G. Edwards, "Optical system-on-a-chip for chemical and biochemical sensing: the platform," in Electro-Optic, Integrated Optic, and Electronic Technologies for Online Chemical Process Monitoring, M. Fallahi, R.J. Nordstrom, and T.R. Todd, eds., Proc. Soc. Photo-Opt. Istrum. Eng. 3537, 0-7 (1999).

6. P. Hlubina and P. Prochazka, "Sensor application of two-mode fiber in the Michelson interferometer configuration," in Interferometry'94: Interferometry Fiber Sensing, E. Udd and R.P. Tatam, eds., Proc. Soc. Photo-Opt. Istrum. Eng. 2341, . 202-211 (1994).

7. L.M. Lechuda, A.T.M. Lenferink, R.P.H. Kooyman, and J. Greve, "Feasibility of evanescent wave interferometer immmunosensors for pesticide detection: Chemical aspects," Sensors and Actuators, B: Chemical B25, 762-765 (1995).

8. R.M. Jenkins, R.W.J. Devereux, and J.M. Heaton, "Novel waveguide Mach-Zehnder interferometer based on multimode interference phenomena," Opt. Commun. 110, $410-424$ (1994). 
9. P.Plizka, and W. Lukasz, "Integrated-optical acoustical sensor," Sensors and Actuators, A: Physical A41, 93-97 (1994).

10. Ch. Fattinger, H. Koller, D. Schlatter, and P. Wehrli, "Difference interferometer: A highly sensitive optical probe for quantification of molecular surface concentration," Biosensors and Bioelectronics 8, 99-107 (1993).

11. W. Ecke, W. Haubenreisser, H. Lehmann, S. Schroeter, G. Schwotzer, and R. Willsch, "Phase-sensitive fibre-optic monoptodes for chemical sensing," Sensors and Actuators, B: Chemical B11, 475-479 (1993).

12. A.A. Boiarski, J.R. Busch, R.S. Brody, R.W. Ridgway, W.P. Altman, and C. Golden, "Integrated optic sensor for measuring aflatoxin-B1 in corn," in Integrated Optics and Microstructures III, M. Tabib-Azar, ed., Proc. Soc. Photo-Opt. Istrum. Eng. 2686, 45$52(1996)$

13. Yu.M. Shirshov, S.V. Svechnikov, A.P. Kiyanovskii, Yu.V. Ushenin, E.F. Venger, A.V. Samoylov, and R. Merker, "A sensor based on the planar-polarization interferometer," Sensors and Actuators, A: Physical A68, 384-387 (1998).

14. Integrated optics, T.Tamir, ed. (Springer-Verlag, Berlin, 1979) p. 47.

15. S.S.Sarkisov, A. Wilkosz, P. Venkateswarlu, "Nonlinear optical waveguides based on polymeric films doped with phthalocyanines," in Physics and Simulation of Optoelectronic Devices IV, Weng W. Chow, Marek Osinsky, eds., Proc. Soc. PhotoOpt. Istrum. Eng. 2693, 523-531 (1996).

16. The Photonics Design \& Applications Handbook (Laurin Publishing Co., Pittsfield, MA , 1999). 
17. E. Nitanai and S. Miyanaga, "Measurements of dispersion properties of refractive indices and absorption coefficients in organic dye-doped thin films by a prism coupling method," Opt. Eng. 35, 900-903 (1996).

18. R. Klein and E.I. Voges, "Integrated optics ammonia sensor," in Advances in fluorescence sensing technology, J.R. Lakiwicz and R.B. Thompson, eds., Proc. Soc. Photo-Opt. Istrum. Eng. 1885, 81-92 (1993).

19. R. Ulrich and R. Torge, "Measurement of thin film parameters with prism coupler," Appl. Opt. 12, 2901-2908 (1973).

20. Handbook of Chemistry and Physics (CRC Press, New York, 1996) p. D-148.

21. P. Caglar and R. Narayanaswamy, "Ammonia-sensitive fibre optic probe utilizing an immobilized spectrophotometric indicator," Analyst 112, 1285-1288 (1987).

22. R.G. Hunsperger, Integrated Optics (Springer-Verlag, Berlin, 1995) p. 25.

\section{Appendix A. List of publications related to the Project}

1. Sergey Sarkisov, Andre Taylor and Putcha Venkateswarlu, "Optical sensors based on single arm thin film waveguide interferometers, NASA Lewis Research Center HBCU Research Conference, Cleveland, Ohio, April 9-10, 1997, Abstracts, p. 10.

2. Sergey Sarkisov, Michael Curley, Darnell Diggs, huaisong Guo, Ronald D. Clark and Grigory Adamovsky, "Optical sensor based on single dual mode polymeric waveguide interferometer", in Polymer Photonic Devices, Bernard Kippelen, Donald D. C. Bradley, editors, Proceedings of SPIE Vol. 3281, pp. 289-299 (1998)

3. Sergey Sarkisov, Michael Curley, Darnell Diggs and Grigory Adomovsky, "Gas sensors based on single-arm waveguide interferometers", in NASA Universty Research Centers. Technical Advances in Aeronautics, Space Sciences and Technology, earth Systems Sciences, 
Global hydrology, and Education. Vol. II, Editors: T.L. Coleman, B. White and S. Goodman, TSI Press, 1998, pp. 257-262.

4. Sergey Sarkisov, Andre Taylor and Putcha Venkateswarlu, "Optical sensors based on single arm thin film waveguide interferometers, NASA Lewis Research Center HBCU Research Conference, Cleveland, Ohio, April 9-10, 1997, Abstracts, P.2.

5. Sergey Sarkisov, Darnell Diggs, Grigory Adamovsky and Michael Curley, "Single-arm double-mode double-order planar waveguide interferometer, Accepted to Applied Optics.

6. Sergey Sarkisov, Andre Taylor, Putcha venkateswarlu, and Grigoruy Adamovsky, "Optical sensor based on single armdual mode polymeric waveguide interferometer, Accepted to SPIE Meeting "Photonics West '98'”

7. Darnell E. Diggs, Sergey S. Sarkisov, Michael J. Curley and Grigory Adamovsky, "Polymer based optical single-arm waveguide interferometer as a chemical sensor", to be published SPIE paper no. $4106 \mathrm{~A}-05$.

8. S. S. Sarkisov, D. Diggs and M. Curley, "Optical sensors based on single-arm thin film waveguide interferometer", HBCUs/OMUs Research Conference Agenda and Abstracts, Cleveland Ohio, P2, 25-26 April, 2000.

9. Sergey Sarkisov, D. Diggs and M. Curley, "Optical sensors based on single-arm thin film waveguide interferometer", HBCUs Research conference Agenda ad Abstracts, 8-9 April, 1998, P1.

Appendix B List of Graduate students supported by NAG3-1956

1. Curtis Banks

2. Darnell Diggs 